TRANSACTIONS OF THE

AMERICAN MATHEMATICAL SOCIETY

Volume 147, February 1970

\title{
GENERALIZED STOCHASTIC INTEGRALS AND EQUATIONS $\left({ }^{1}\right)$
}

\author{
BY \\ DONALD A. DAWSON
}

1. Introduction. In his fundamental memoir [7] K. Itô introduced an important class of stochastic differential equations which are now known as Itô equations. These equations are based on his definitions of stochastic integrals with respect to Brownian motion and random measures with independent values. The importance of these equations is due to the fact that a large class of Markov processes in $R^{n}$ can be represented as solutions of such equations. A thorough account of Itô's work and various extensions of it has been given by A. V. Skorohod [18]. It is also possible to represent certain other Markov processes of finite order as solutions of vector Itô equations. In this paper we consider an extension of Itô integrals to integrals with respect to generalized processes with independent values in the sense of I. M. Gel'fand and N. Ja. Vilenkin [5]. We do not consider the most general case which would involve integration with respect to generalized processes with independent values defined in $R^{n}, n \geqq 1$ (cf. K. Itô [7] and A. V. Skorohod [18] for random measures) but restrict ourselves to the case $n=1$. The relation between the generalized Itô integral and the multiplication of (Schwartz) distributions is discussed. This involves a study of $\mu$-transformations of $\mathscr{D}^{\prime}$, the space of distributions, where $\mu$ is a measure on $\mathscr{D}^{\prime}$. Following the example of K. Itô we then discuss stochastic differential equations defined in terms of these stochastic integrals and prove several existence theorems.

It is shown that the solutions of generalized Itô equations with local coefficients have local splitting $\sigma$-fields and are weakly Markov of finite order. Generalized Itô equations may also be considered in connection with $P$. Levy's problem of the representation of stochastic processes in terms of differential innovation processes $[11, \S 2.7]$.

2. Preliminaries on generalized processes. The theory of generalized stochastic processes are thoroughly studied in I. M. Gel'fand and N. Ja. Vilenkin [5] and in $\mathrm{X}$. Fernique [3]. In this section we review those parts of the theory which are relevant to this paper.

A generalized stochastic process $X$ is a mapping $\phi \leadsto X(\phi)$ of $\mathscr{D}$, the space of

Received by the editors March 22, 1969.

$\left({ }^{1}\right)$ This research was supported in part by the Summer Research Institute of the Canadian Mathematical Congress.

Copyright (C) 1970, American Mathematical Society 
$C^{\infty}$-functions with compact support in $R^{1}$, into the family of random variables on a probability measure space $(\Omega, \mathscr{F}, P)$ which satisfies

(2.1) $X(\alpha \phi+\beta \psi)=\alpha X(\phi)+\beta X(\psi)$ for $\alpha, \beta \in R^{1}$, and

(2.2) if $\phi_{n} \rightarrow 0$ in $\mathscr{D}$ with the usual topology, then $X\left(\phi_{n}\right) \rightarrow 0$ in probability.

If for each $\omega, \phi \leadsto X(\phi)(\omega)$ is linear and continuous on $\mathscr{D}, X$ is said to be a random distribution. If $X$ is a random distribution, then a measure is induced on $\left(\mathscr{D}^{\prime}, \mathscr{B}\left(\mathscr{D}^{\prime}\right)\right)$ where $\mathscr{B}\left(\mathscr{D}^{\prime}\right)$ is the $\sigma$-field of Borel subsets of $\mathscr{D}^{\prime}$. Because of the nuclearity of $\mathscr{D}$, the fundamental theorem of R. A. Minlos [5, Chapter 4, Theorem 2.3] yields the following.

THEOREM 2.1 Every generalized random process has a modification which is a random distribution.

Proof. See Fernique [3, Proposition III, 4.2(a)].

The following result is easy to verify.

THEOREM 2.2 The derivative of a random distribution is a random distribution.

Proof. See I. M. Gel'fand and N. Ja. Vilenkin [5, p. 246]. (All derivatives are derivatives in the sense of distributions and are denoted by $d f / d t, \dot{f}$ or $D f$.)

The mean of a generalized process is defined (if it exists) by

$$
M(\phi) \equiv E(X(\phi)), \quad \phi \in \mathscr{D} .
$$

The mean is a distribution.

The correlation functional of $X$ is defined (if it exists) by

$$
\Gamma(\phi, \psi) \equiv E[X(\phi) \cdot X(\psi)] \text { for } \phi, \psi \in \mathscr{D} \text {. }
$$

The kernels theorem implies that $\Gamma(\phi, \psi)=K(\phi \otimes \psi)$ for some $K \in \mathscr{D}^{\prime}\left(R^{1} X R^{1}\right)$. A process for which $\Gamma(\phi, \phi)$ exists for all $\phi$ is said to be a second order generalized process. Note that $\Gamma(\cdot, \cdot)$ is a positive definite bilinear form. For second order generalized processes Fernique has proved the following fundamental convergence theorem.

THEOREM 2.3. Let $\left\{X_{n}\right\}$ be a sequence of second order random distributions such that for every $\phi \in \mathscr{D}, E\left(\left(X_{n}-X_{m}\right)(\phi)\right)^{2} \rightarrow 0$ as $n, m \rightarrow \infty$. Then there exists a subsequence $\left\{X_{n_{k}}\right\}$ which converges almost surely. The different limits are equal, almost surely.

Proof. X. Fernique [3, Theorem III, 7.2].

$X$ is said to be a generalized process with independent values at every point if the random variables $X(\phi)$ and $X(\psi)$ are mutually independent random variables whenever Spt $(\phi) \cap \operatorname{Spt}(\psi)=\varnothing$. The following theorem characterizes the correlation functional of a process with independent values at every point.

THEOREM 2.4. The correlation functional $\Gamma(\cdot, \cdot)$ of a process with independent values at every point is given by

$$
\Gamma(\phi, \psi)=\iint \sum_{j, k=0}^{\infty} r_{j, k}(x, t) \phi^{(j)}(x) \psi^{(k)}(x) d \mu(x, t)
$$


where $\phi^{(j)}(x)=d^{j} \phi(x) / d x^{j}, \mu$ is a positive measure, the functions $r_{j, k}(\cdot, \cdot)$ are $\mu$-square integrable and only a finite number of the $r_{j, k}(\cdot, \cdot)$ are nonzero on any given bounded set.

Proof. See Fernique [3, Theorem IV.1.3] (cf. I. M. Gel'fand and N. Ja. Vilenkin [5, III, Theorem 9]).

Note that if $T$ is a differential operator of finite order, then the correlation functional of $T X$ satisfies $\Gamma_{T X}(\phi, \psi)=\Gamma_{X}(T \phi, T \psi)$. Many different generalized stochastic processes with independent values at every point can have the same correlation functional. A rather large class of processes with independent values at every point is characterized in terms of the characteristic functional in I. M. Gel'fand and N. Ja. Vilenkin [5, III, 4].

Let $\mathscr{D}^{m}$ be the set of functions with compact support and $m$ continuous derivatives. The space of distributions which is the dual of $\mathscr{D}^{m}$ is denoted by $\mathscr{D}^{\prime m}$ and its elements are called distributions of order $\leqq m$. A distribution which is of order $\leqq m$ but not of order $\leqq m-1$ is said to be of order $m$. R. M. Dudley has proved the following theorem.

THEOREM 2.5. If for every $\phi \in \mathscr{D}$

$$
\sup _{h} E\left(X\left(\tau_{h} \phi\right)\right)^{2}<\infty
$$

where $\left(\tau_{h} \phi\right)(t) \equiv \phi(t-h)$, then there is a positive integer $m$ such that the measure induced by $X$ is concentrated on the set of distributions which are of the form $D^{m} f$ for some continuous function $f$.

Proof. See Dudley [2, Theorem 3.1].

The Sobolev space $H^{2, m}(U)$ is the set of distributions in an open set $U$ such that the distribution derivatives of order $\leqq m$ belong to $L^{2}(U)$. The space $H^{2, m}(U)$ is a normed space with norm

$$
\|u\|_{2, m}=\left(\sum_{0 \leqq \alpha \leqq m} \int_{U}\left(D^{\alpha} u(x)\right)^{2} d x\right)^{1 / 2} .
$$

$H_{0}^{2, m}(U)$ is the closure of $\mathscr{D}^{m}(U)$, the set of functions in $\mathscr{D}^{m}$ with compact support in $U$, in $H^{2, m}(U) . H^{\infty, m}(U)$ is the set of distributions defined in an open set $U$ such that the distribution derivatives of order $\leqq m$ belong to $L^{\infty}(U)$. The space $H^{\infty, m}(U)$ is a normed space with norm

$$
\|u\|_{\infty, m}=\sum_{0 \leqq \alpha \leqq m}\left\|D^{\alpha} u\right\|_{\infty} .
$$

THEOREM 2.6. $H^{2, m}(U)$ and $H_{0}^{2, m}(U)$ are Hilbert spaces.

Proof. See F. Treves [20, Proposition 31.1].

Let $H^{2,-m}(U)$ be the space of distributions in $U$ which are equal to a finite sum of derivatives of order $\leqq m$ of functions in $L^{2}(U)$. 
THEOREM 2.7 If $m$ is a positive integer, then there is a canonical linear bijection of $H^{2,-m}(U)$ onto the dual of $H_{0}^{2, m}(U)$.

Proof. See F. Treves [20, Proposition 31.3].

For $m$ positive or negative $H_{\mathrm{loc}}^{2, m}$ is the set of distributions whose restrictions to any relatively compact open set $U$ lie in $H^{2, m}(U)$.

A fundamental system of neighbourhoods of 0 for the usual topology, $\mathscr{T}_{b}$; in $\mathscr{D}^{\prime}$ are of the form

$$
V(B, \varepsilon)=\{T:|T(\phi)| \leqq \varepsilon \text { for } \phi \in B\}
$$

where $\varepsilon>0$ and $B$ is a bounded set in $\mathscr{D}$. The weak topology $\mathscr{T}_{w}$ is given by the fundamental system of neighbourhoods of the form

$$
V\left(\varepsilon, \phi_{1}, \ldots, \phi_{n}\right)=\bigcap_{j=1}^{n}\left\{T:\left|T\left(\phi_{j}\right)\right|<\varepsilon\right\}
$$

where $\varepsilon>0$ and $\phi_{1}, \ldots, \phi_{n}$ are in $\mathscr{D}$. Let $\mathscr{B}_{b}, \mathscr{B}_{w}, \hat{\mathscr{B}}_{b}, \hat{\mathscr{B}}_{w}$ be the respective Baire and Borel sets and let $\mathscr{B}\left(\mathscr{D}^{\prime}\right)$ be the $\sigma$-field of subsets of $\mathscr{D}^{\prime}$ generated by the family of sets of the form $\{T: T(\phi) \in B\}$ where $\phi \in \mathscr{D}$ and $B \in \mathscr{B}\left(R^{1}\right)$. Let $\mathscr{B}\left(\mathscr{D}^{\prime}, I\right)$ be the sub- $\sigma$-field of $\mathscr{B}\left(\mathscr{D}^{\prime}\right)$ generated by the family of sets of the form $\{T: T \phi \in B\}$ where $B \in \mathscr{B}\left(R^{1}\right), \phi \in \mathscr{D}$ and Spt $(\phi) \subset I$. If $X$ is a generalized process defined on the probability measure space $(\Omega, \mathscr{F}, P)$ let $\mathscr{F}_{I}^{X}$ be the $\sigma$-field generated by the random variables $X(\phi)$, Spt $(\phi) \subset I$.

THEOREM 2.8. $\hat{\mathscr{B}}_{w}=\mathscr{B}_{w}=\hat{\mathscr{B}}_{b}=\mathscr{B}_{b}=\mathscr{B}$.

Proof. See Ju. V. Prohorov [14].

THEOREM 2.9. The sets $C$, the set of continuous functions, $\mathscr{D}^{\prime m}$ and $H^{2,-m}$ are Borel subsets of $\mathscr{D}^{\prime}$.

Proof. See Fernique [3, III. 3].

Given a compact subset $K$ of $R^{1}$, let $\mathscr{D}_{K}=\{\phi: \phi \in \mathscr{D}$, Spt $(\phi) \subset K\}$. Then there exists a natural measurable mapping $\pi:\left(\mathscr{D}^{\prime}, \mathscr{B}\left(\mathscr{D}^{\prime}\right)\right) \rightarrow\left(\mathscr{D}_{K}^{\prime}, \mathscr{B}\left(\mathscr{D}_{K}^{\prime}\right)\right)$. Given a measure $\mu$ on $\left(\mathscr{D}^{\prime}, \mathscr{B}\left(\mathscr{D}^{\prime}\right)\right)$ then the measure $\mu_{K}$ on $\left(\mathscr{D}_{K}^{\prime}, \mathscr{B}\left(\mathscr{D}_{K}^{\prime}\right)\right)$ which is induced by $\pi$ is called the $K$-marginal of $\mu$.

THEOREM 2.10. In order that a system $\left\{\mu_{K}\right\}$ of probability measures on $\left(\mathscr{D}_{K}^{\prime}, \mathscr{B}\left(\mathscr{D}_{K}^{\prime}\right)\right)$ where $K$ runs through the set of compact sets be a system of $K$-marginals of a random distribution, it is necessary and sufficient that for every pair $K_{1}, K_{2}$ and all $\phi \in \mathscr{D}_{K_{1}} \cap \mathscr{D}_{K_{2}}$,

$$
\mu_{K_{1}}\left(\left\{T: T \in \mathscr{D}_{K_{1}}^{\prime},|T(\phi)| \leqq 1\right\}\right)=\mu_{K_{2}}\left(\left\{T: T \in \mathscr{D}_{K_{2}}^{\prime},|T(\phi)| \leqq 1\right\}\right) .
$$

Proof. See Fernique [3, Proposition III. 4.3]. 
THEOREM $2.11\left({ }^{2}\right) . \mathscr{D}^{\prime}$ is a Lusin space.

Proof. See L. Schwartz [17].

THEOREM 2.12. For any probability measure $P$ on $\left(\mathscr{D}^{\prime}, \mathscr{B}\left(\mathscr{D}^{\prime}\right)\right)$ and any sub- $\sigma-f i e l d$, $\mathscr{A}$, of $\mathscr{B}\left(\mathscr{D}^{\prime}\right)$ there exists a regular conditional probability with respect to $\mathscr{A}$.

Proof. Since $\mathscr{D}^{\prime}$ is Lusin, there is a finer topology $\mathscr{T}^{1} \supset \mathscr{T}_{b}$ such that $\left(\mathscr{D}^{\prime}, \mathscr{T}^{1}\right)$ is Polish. Hence $\left(\mathscr{D}^{\prime}, \mathscr{B}\left(\mathscr{T}^{1}\right)\right)$ satisfies the conditions of the well-known theorem of M. Jirina [9] which implies the existence of regular conditional probabilities.

THEOREM 2.13. If the generalized stochastic process $X$ satisfies $E[X(\phi)]^{2} \leqq k\|\phi\|_{2, m}^{2}$ for all $\phi$ with support in a compact set $K$, then the $K$-marginal measure can be concentrated on $H^{2,-(m+2)}$.

Proof. See Gel'fand and Vilenkin [5, 329].

THEOREM 2.14. Let $\mathscr{B}_{t}^{*}=\bigcap_{\varepsilon>0} \mathscr{B}\left(\mathscr{D}^{\prime},[t-\varepsilon, t+\varepsilon]\right)$ and let $\mathscr{B}_{\infty} \equiv \bigvee_{t} \mathscr{B}_{t}^{*}$. Then $\mathscr{B}_{\infty} \nsubseteq \mathscr{B}\left(\mathscr{D}^{\prime}\right)$.

Proof. Consider the measures $\mu$ and $\nu$ on $\mathscr{B}\left(\mathscr{D}^{\prime}\right)$ induced by the first derivative of the Poisson process and the identically zero process respectively. Now these two measures agree on $\mathscr{B}_{t_{1}}^{*} \vee \cdots \vee \mathscr{B}_{t_{n}}^{*}$ for $\left(t_{1}, \ldots, t_{n}\right) \in R^{n}$ and hence they agree on $\mathscr{B}_{\infty}$. Since $\mu \neq \nu$ on $\mathscr{B}\left(\mathscr{D}^{\prime}\right)$ it follows that $\mathscr{B}_{\infty} \neq \mathscr{B}\left(\mathscr{D}^{\prime}\right)$. On the other hand it is obvious that $\mathscr{B}_{\infty} \subset \mathscr{B}\left(\mathscr{D}^{\prime}\right)$.

We will also require the following theorem.

THEOREM 2.15. Consider the system of differential equations

$$
D T_{i}+\sum_{j=1}^{M} A_{i j}(t) T_{j}=S_{i}, \quad i=1, \ldots, M \text { where } D T_{i}=\frac{d}{d t} T_{i},
$$

$A_{i, j}(\cdot)$ are $C^{\infty}$-functions and $S^{i} \in \mathscr{D}^{\prime}, i=1, \ldots, M$. If $S_{i}=0, i=1, \ldots, M$ then the only solutions are $C^{\infty}$-functions. For any $\left\{S_{i}\right\}$ there exist solutions to the system and the difference between any two solutions is a solution of the homogeneous system.

Proof. See Friedman [4, Theorem 36].

3. Stochastic integrals. I. Throughout the remainder of this paper $X$ denotes a second order generalized stochastic process with independent values at every point, zern mean and correlation functional $\Gamma(\cdot, \cdot)$. Since $\Gamma$ is a nonnegative quadratic form (Theorem 2.4) on $\mathscr{D}$, we can complete $\mathscr{D}$ with respect to $\Gamma$ to obtain a Hilbert space $H_{\Gamma}$ with inner product $\Gamma$. Instead of taking the most general form of $\Gamma$ given by Theorem 2.4 we assume that $\Gamma(\cdot, \cdot)$ is of the form

$$
\Gamma(\phi, \psi)=\int \sum_{j, k=0}^{N} r_{j k}(x) \phi^{(j)}(x) \psi^{(k)}(x) d x
$$

${ }^{2}$ ) I would like to thank Professors J. R. Choksi and K. N. Gowrisankaran for their communication of the results of [17] and their assistance with the proof of 2.12. 
where $N<\infty$ and the functions $r_{j k}(\cdot)$ are in $L^{\infty}\left(R^{1}\right)$.

The set of functions

$$
\left\{f: \sum_{j, k=0}^{N} \int r_{j k}(x) f^{(j)}(x) f^{(k)}(x) d x<\infty\right\}
$$

modulo $N_{\Gamma}=\{f: \Gamma(f, f)=0\}$ can be identified with an appropriate subspace of $H_{\Gamma}$ (N.B. Derivatives always refer to distribution derivatives.)

Proposition 3.1. If $\Gamma(\cdot, \cdot)$ is of the form (3.1), then $H_{0}^{2, N} / N_{\Gamma} \subset H_{\Gamma}$.

Proof.

$$
\begin{aligned}
\|f\|_{\Gamma}^{2} & =\sum_{j, k=0}^{N} \int_{-\infty}^{\infty} r_{j, k}(x) f^{(j)}(x) f^{(k)}(x) d x \\
& \leqq \sum_{j, k=0}^{N}\left\|r_{j, k}\right\|_{\infty} \int_{-\infty}^{+\infty}\left|f^{(j)}(x) f^{(k)}(x)\right| d x \\
& \leqq \sum_{j, k=0}^{N}\left\|r_{j, k}\right\|_{\infty}\left(\int_{-\infty}^{\infty}\left(f^{(j)}(x)\right)^{2} d x\right)^{1 / 2}\left(\int_{-\infty}^{\infty}\left(f^{(k)}(x)\right)^{2} d x\right)^{1 / 2}
\end{aligned}
$$

by Schwarz's inequality

$$
\leqq \sum_{j, k=0}^{N}\left\|r_{j, k}\right\|_{\infty}\|f\|_{2, N}^{2}
$$

The proof is completed by noting that $\mathscr{D}$ is dense in $H_{0}^{2, N}$. (We do not consider here the question of when $H_{\Gamma}=H_{0}^{2, N}$ but it is related to Gårding's inequality [22, VI. 8].)

The mapping $\phi \leadsto X(\phi)$ of $\mathscr{D}$ into $L^{2}(\Omega, \mathscr{F}, P)$ extends naturally to an isometry of $H_{\Gamma}$ onto a closed subspace of $L^{2}(\Omega, \mathscr{F}, P)$ such that

$$
E(X(f) \cdot X(g))=\Gamma(f, g) .
$$

This extension is called the second order extension of $X$.

EXAmple 3.1. Let $X$ be the first derivative, $\dot{B}$, of the Brownian motion process $B$. Then $\dot{B}$ is a second order generalized process with independent values at every point and correlation functional

$$
\Gamma(\phi, \psi)=\int_{-\infty}^{\infty} \phi(x) \psi(x) d x .
$$

$\dot{B}$ is usually known as the Gaussian white noise generalized process. The Itô integral $\int_{-\infty}^{\infty} f(t) d B(t)$ is a linear isometry of $L^{2}\left(R^{1}\right)$ into $L^{2}(\Omega, \mathscr{F}, P)$ which is defined for a simple function $f(\cdot)=\sum_{i=1}^{n} c_{i} \chi_{\left[a_{i}, b_{i}\right)}$ by

$$
\int_{-\infty}^{\infty} f(t) d B(t)=\sum_{i=1}^{n} c_{i}\left(B\left(b_{i}\right)-B\left(a_{i}\right)\right)
$$


It is well known that if $\phi \in \mathscr{D}$, then

$$
\begin{aligned}
\int_{-\infty}^{\infty} \phi(t) d B(t) & =-\int_{-\infty}^{\infty} B(t) \dot{\phi}(t) d t \quad \text { a.e. } \\
& =-B(\dot{\phi})=\dot{B}(\phi) .
\end{aligned}
$$

Hence the usual stochastic integral is the second order extension of the Gaussian white noise process $\dot{B}$.

In the study of stochastic differential equations it is also necessary to consider the indefinite integral

$$
\int^{t} f(t) d B(t)=\int \chi_{(-\infty, t]}(s) f(s) d B(s)+c
$$

where $c$ is an arbitrary random constant. In the general case with $X$ playing the role of $\dot{B}$ the function $\chi_{(-\infty, t]}$ may not belong to $H_{\Gamma}$ so that we may not be able to define the indefinite integral in this manner. However, in this case we first define the product $f X$ as a distribution and then consider the primitive, $\int f(t) X$, of $f(t) X$ as a distribution. We now proceed to do this.

If $\Phi: \mathscr{D} \rightarrow H_{\Gamma}$ is a continuous linear mapping, then so is the composition

$$
\mathscr{D} \stackrel{\Phi}{\longrightarrow} H_{\Gamma} \stackrel{X}{\longrightarrow} L_{2}(\Omega, \mathscr{F}, P) \text {. }
$$

It is easy to verify that this yields a generalized stochastic process and hence there is a modification which is a second order random distribution with correlation functional

$$
\Gamma(\phi, \psi)=\int_{-\infty}^{\infty} \sum_{j, k=0}^{N} r_{j, k}(x) \Phi(\phi)^{(j)}(x) \Phi(\psi)^{(k)}(x) d x .
$$

Following Fernique we call $X \Phi$ the second order transformation (or $P$-transformation) of $X$ by $\Phi$. We next apply this to obtain $P$-products.

For fixed $f$ consider the linear mapping $\Phi$ from $\mathscr{D}$ to $H_{\Gamma}$ defined by $\phi \leadsto f \cdot \phi$.

Proposition 3.3 If assumption 3.1 is satisfied and if $f \in H_{0}^{N, 2}$, then the mapping $\Phi$ is continuous.

Proof.

$$
\begin{aligned}
\|f \phi\|_{\mathrm{r}}^{2} & =\sum_{j, k=0}^{N} \int_{-\infty}^{\infty} r_{j, k}(x)(f \phi)^{(j)}(x)(f \phi)^{(k)}(x) d x \\
& =\sum_{j, k=0}^{N} \sum_{i=0}^{j} \sum_{l=0}^{k} \int_{-\infty}^{\infty} r_{j, k}(x)\left(\begin{array}{l}
j \\
i
\end{array}\right)\left(\begin{array}{l}
k \\
l
\end{array}\right) f^{(i)}(x) \phi^{(j-i)}(x) f^{(l)}(x) \phi^{(k-l)}(x) d x \\
& \leqq\|\phi\|_{\infty, N}^{2} \sum_{j, k=0}^{N} \sum_{i=0}^{j} \sum_{l=0}^{k} \int_{-\infty}^{\infty}\left|r_{j, k}(x)\left(\begin{array}{l}
j \\
i
\end{array}\right)\left(\begin{array}{l}
k \\
l
\end{array}\right) f^{(i)}(x) f^{(l)}(x)\right| d x \\
& \leqq \alpha\|\phi\|_{\infty, N}^{2}\|f\|_{2, N}^{2} \text { for an appropriate constant } \alpha .
\end{aligned}
$$

Therefore if $\phi_{n} \rightarrow 0$ in $\mathscr{D}$, then $\left\|f \phi_{n}\right\|_{\Gamma} \rightarrow 0$ and the proof is complete. 
Therefore the second order transformation of $X$ by the mapping $\phi \leadsto f \phi$ gives us a $P$-transformation which we call the $P$-product, $X f$, of $f$ and $X$. If $f \in \mathscr{D}$, then for almost every $\omega, X f(\omega)$ is the product of $f$ with the distribution $X(\omega)$ in the usual sense of the theory of distributions. In addition $X f(\phi)$ is the stochastic integral of $\phi$ with respect to $X f$. If assumption 3.1 is satisfied, then Proposition 3.1 implies that $E\left((X \phi)^{2}\right) \leqq \alpha\|\phi\|_{2, N}^{2}$ which in turn implies that the $K$-marginal measures can be concentrated on $H^{2,-(N+2)}$ (Theorem 2.13). Hence the usual distribution product $X(\omega) f$ is defined for almost every $\omega$ only if $f \in H_{0}^{2, N+2}$. Thus we would not expect to be able to multiply $X$ by an arbitrary $f \in H_{0}^{2, N}$. The fact that we have defined such a $P$-product can perhaps be explained by the fact that if $f_{n} \rightarrow f$ in $H_{0}^{2, N}, f_{n} \in \mathscr{D}$, $n=1,2, \ldots$, then for each $\phi \in \mathscr{D}, X f_{n}(\phi) \rightarrow X f(\phi)$ in $L^{2}$ but not necessarily almost surely. However, by Theorem 2.3 there is a subsequence $X f_{n_{k}} \rightarrow X f$ in $\mathscr{D}^{\prime}$ almost surely.

REMARK 3.1. If $f \in H_{\mathrm{loc}}^{2, N}$, then by a minor modification of the above argument we can define $X f$ restricted to any relatively compact open set.

REMARK 3.2. L. Schwartz [15] has shown that it is impossible to define multiplication for all pairs of distributions in such a way as to maintain the usual rules of differentiation.

The primitive of the random distribution $X f$ is denoted by $\int^{t} X f$, that is, if $\phi \in \mathscr{D}$ then $\left(\int^{t} X f\right)(\dot{\phi}) \equiv-X f(\phi)$. If we fix $\phi_{0} \in \mathscr{D}$ which is not of the form $\dot{\psi}$ for $\psi \in \mathscr{D}$, then we can allow $\left(\int^{t} X f\right)\left(\phi_{0}\right)$ to be an arbitrary random constant.

Proposition 3.4. For any $f \in H_{0}^{2, N}$ and any $\phi \in \mathscr{D}, E(X f(\phi))=0$, that is, the mean of $X f$ is zero.

Proof. By definition there exists $\psi_{n} \in \mathscr{D}, n=1,2, \ldots$, such that $\psi_{n} \rightarrow f$ in $H_{0}^{2, N}$. But $E\left(X \psi_{n}(\phi)\right)=E\left(X\left(\psi_{n} \phi\right)\right)=0$ since $X$ has zero mean. By Proposition 3.3. $X \psi_{n}(\phi) \rightarrow X f(\phi)$ in $L^{2}(\Omega, \mathscr{F}, P)$ which implies that $X \psi_{n}(\phi) \rightarrow X f(\phi)$ in $L^{1}(\Omega, \mathscr{F}, P)$. Therefore $E(X f(\phi))=0$.

Proposition 3.5. If $f \in H_{\mathrm{loc}}^{\infty, N}$ then the $K$-marginals of the measure induced by $X f$ on $\mathscr{D}^{\prime}$ can be concentrated on $H_{\mathrm{loc}}^{2,-(N+2)}$, that is, Xf can be concentrated on $H_{\mathrm{loc}}^{2,-(N+2)}$.

Proof. Consider a relatively compact open set $U$, and assume that $\operatorname{Spt}(\phi) \subset \bar{U}$. Then

$$
\begin{aligned}
E\left((X f(\phi))^{2}\right) & =\|f \phi\|_{\Gamma}^{2} \\
& =\sum_{j, k=0}^{N} \int_{-\infty}^{\infty} r_{j, k}(x)(f \phi)^{(j)}(f \phi)^{(k)} d x \\
& =\sum_{j, k=0}^{N} \sum_{i=0}^{j} \sum_{l=0}^{k} \int_{-\infty}^{\infty}\left(\begin{array}{l}
j \\
i
\end{array}\right)\left(\begin{array}{l}
k \\
l
\end{array}\right) f^{(i)} \phi^{(j-i)} f^{(l)} \phi^{(k-l)} r_{j, k} d x \\
& \leqq\left\|\left.f\right|_{U}\right\|_{\infty, N}^{2} \sum_{j, k=0}^{N} \sum_{i=0}^{j} \sum_{l=0}^{k} \int_{-\infty}^{\infty}\left(\begin{array}{l}
j \\
i
\end{array}\right)\left(\begin{array}{l}
k \\
l
\end{array}\right)\left|r_{j, k}(x)\right|\left|\phi^{(i)}(x) \cdot \phi^{(l)}(x)\right| d x \\
& \leqq \alpha\left\|\left.f\right|_{U}\right\|_{\infty, N}^{2}\|\phi\|_{2, N}^{2} .
\end{aligned}
$$


The result then follows from Theorem 2.13.

4. Stochastic integrals. II. In this section the notion of stochastic integral is extended to the case of random integrands. As in the previous section this requires a consideration of $P$-transformations of $\mathscr{D}^{\prime}$ and in this section these transformations will in general be nonlinear. We will first review the approach of $\mathrm{K}$. Itô in the case of Example 3.1 (refer to J. L. Doob [1, IX, 5] for the details).

Let $V=L^{2}\left(R^{1} \times \Omega, \mathscr{B}\left(R^{1}\right) \times \mathscr{F}, d t \times P\right)$ and let

$$
\|f(t, \omega)\|_{2} \equiv\left[\int_{-\infty}^{\infty} E\left([f(t, \omega)]^{2}\right) d t\right]^{1 / 2}
$$

Let $V^{M}=\left\{f(\cdot, \cdot): f(\cdot, \cdot) \in V ; \forall s, f(s, \cdot)\right.$ is $\mathscr{F}_{(-\infty, s]}$-measurable $\}$. A $(t, \omega)$-step function is a function of the form

$$
\begin{aligned}
f(t, \omega) & =0, & & t<a_{1}, \\
& =f_{j}(\omega), & & a_{j} \leqq t<a_{j+1}, \quad j<n, \\
& =0, & & a_{n} \leqq t,
\end{aligned}
$$

where $a_{1}<a_{2}<\cdots<a_{n}$ and $f_{j}(\cdot)$ is $\mathscr{F}_{\left(-\infty, a_{j}\right]}$-measurable. It can be shown that the $(t, \omega)$-step functions are dense in $V^{M}$. For such a $(t, \omega)$-step function the stochastic integral is defined by

$$
\int_{-\infty}^{\infty} f(t, \omega) d B(t)=\sum_{j=0}^{n-1} f_{j}(\omega)\left(B\left(a_{j+1}\right)-B\left(a_{j}\right)\right) .
$$

Then the mapping $f \leadsto \int_{-\infty}^{\infty} f(t, \omega) d B(t)$ can be extended to an isometry from $V^{M}$ to $L^{2}(\Omega, \mathscr{F}, P)$.

Now let $X$ be a generalized process with independent values and correlation functional $\Gamma(\cdot, \cdot)$ of the form 3.1. A function $f: R^{1} \times \Omega \rightarrow R^{1}$ is said to be $\mathscr{F}^{x_{-}}$ progressively measurable if for each $t \in R^{1}$, the mapping $f:(-\infty, t] \times \Omega \rightarrow R^{1}$ is measurable with respect to $\mathscr{B}(-\infty, t] \times \mathscr{F}_{(-\infty, t]}^{\mathrm{X}}$.

Let

$$
W^{N}=\left\{f: f \text { is } \mathscr{F}^{\mathrm{x}} \text {-progressively measurable, } f \in H_{0}^{2, N} \text { a.s., } E\left(\|f\|_{2, N}^{2}\right)<\infty\right\},
$$

and let

$$
\|f\|_{2, N, t}^{2} \equiv E\left[\int_{-\infty}^{t} \sum_{0 \leqq \alpha \leqq N}\left(\frac{d^{\alpha} f(x)}{d x^{\alpha}}\right)^{2} d x\right] .
$$

For $a \in R^{1}$ let

$$
\begin{aligned}
W_{a}=\{f: \operatorname{Spt}(f) & \subset[a, \infty) \text { a.s., } f(t, \cdot) \in H_{0}^{2, N} \text { a.s., } \\
& \left.f(\cdot, \cdot) \text { is } \mathscr{B}\left(R^{1}\right) \times \mathscr{F}_{(-\infty, a]}^{X} \text {-measurable, } E\left(\|f\|_{2, N}^{2}\right)<\infty\right\} .
\end{aligned}
$$


We define the inner products

$$
\begin{aligned}
\langle f(\cdot, \cdot), g(\cdot, \cdot)\rangle_{\Gamma} & =E\left[\int_{-\infty}^{\infty} \sum_{j, k=0}^{N} r_{j, k}(x) f^{(j)}(x, \omega) g^{(k)}(x, \omega) d x\right], \\
\langle f(\cdot, \cdot), g(\cdot, \cdot)\rangle_{\Gamma, s} & =E\left[\int_{-\infty}^{s} \sum_{j, k=0}^{N} r_{j, k}(x) f^{(j)}(x, \omega) g^{(k)}(x, \omega) d x\right]
\end{aligned}
$$

in $W^{N}$. The spaces $W^{N}$ and $W_{a}$ completed with respect to $\langle\cdot, \cdot\rangle_{\Gamma}$ are denoted by $W^{*}, W_{a}^{*}$ respectively. It is easy to verify that $W^{*} / N^{*}$ is a Hilbert space where $N^{*} \equiv\left\{f: f \in W^{*},\langle f, f\rangle_{\Gamma}=0\right\}$. Let $W^{* 0}$ denote the set of $W^{*}$-functions of compact support.

Let

$$
\begin{array}{r}
\hat{W}_{a}=\left\{f: f \in W_{a}^{*}, f=\sum_{i=1}^{m} \chi_{A_{i}} f_{i}, \operatorname{Spt}\left(f_{i}\right) \subset[a, \infty), A_{i} \in \mathscr{F}_{(-\infty, a]}^{X}, i=1, \ldots, m,\right. \\
\left.A_{i} \cap A_{j}=\varnothing \text { if } i \neq j, f_{i} \in H_{\Gamma}, i=1, \ldots, m\right\} .
\end{array}
$$

We now proceed to define the $P$-product $f X$ for $f \in W^{*}$. In order to do this we begin by defining the stochastic integral $X(f)$ for $f \in \hat{W}_{a}, a \in R^{1}$. For $f \in \hat{W}_{a}$, $f=\sum_{i=1}^{m} \chi_{A_{i}} f_{i}, A_{i} \in \mathscr{F}_{(-\infty, a]}^{X}, i=1, \ldots, m, A_{i} \cap A_{j}=\varnothing$ if $i \neq j, f_{i} \in H_{\Gamma}, i=1, \ldots, m$, we define $X(f)$ by

$$
X(f)=\sum_{i=1}^{m} \chi_{A_{i}} X\left(f_{i}\right)
$$

where $X\left(f_{i}\right)$ is the second order extension of $X(\cdot)$ which is defined in $\S 3$.

Proposition 4.1. The mapping from $\hat{W}_{a}$ to $L^{2}(\Omega, \mathscr{F}, P)$ defined by equation 4.1 preserves inner products.

Proof. Let $f=\sum_{i=1}^{m} \chi_{A_{i}} f_{i}$ and $g=\sum_{i=1}^{n} \chi_{B_{l}} g_{i}$. Since $X$ has independent values, it follows that $\chi_{A_{i}}$ and $X\left(f_{i}\right)$ are independent and $\chi_{B_{i}}$ and $X\left(g_{i}\right)$ are independent. Therefore

$$
\begin{aligned}
E(X(f) \cdot X(g)) & =E\left[\sum_{i=1}^{m} \sum_{j=1}^{n} \chi_{A_{i} \cap B_{j}} \cdot\left(X\left(f_{i}\right) \cdot X\left(g_{j}\right)\right)\right] \\
& =\sum_{i=1}^{m} \sum_{j=1}^{n} P\left(A_{i} \cap B_{j}\right) E\left(X\left(f_{i}\right) \cdot X\left(g_{j}\right)\right) \\
& =\sum_{i=1}^{m} \sum_{j=1}^{n} P\left(A_{i} \cap B_{j}\right) \int_{-\infty}^{\infty} \sum_{k, l=0}^{N} r_{k, l}(x) f_{i}^{(k)}(x) g_{j}^{(l)}(x) d x \\
& =E\left[\int_{-\infty}^{\infty} \sum_{k, l=0}^{N} r_{k, l}(x) f^{(k)}(x, \omega) g^{(l)}(x, \omega) d x\right] \\
& =\left\langle\sum_{i=1}^{m} \chi_{A_{i}} f_{i}, \sum_{j=1}^{m} \chi_{B_{j} g_{j}}\right\rangle_{\Gamma}
\end{aligned}
$$


Proposition 4.2. $\hat{W}_{a}$ is dense in $W_{a}^{*}$.

Proof. Let $f \in W_{a}^{*}$ and let $\mathscr{G}$ be the $\sigma$-field of subsets of $\Omega$ generated by $f$ considered as a $H_{\Gamma}$-valued random variable. It can be shown that $\mathscr{G}$ is generated by sets of the form $\left\{\omega:(f(\omega), \phi) \in B, B \in \mathscr{B}\left(R^{1}\right), \phi \in \mathscr{D}\right.$, Spt $\left.(\phi) \subset[a, \infty)\right\}$. Since Fubini's theorem implies that $(f, \phi)$ is $\mathscr{F}_{(-\infty, a]}^{X}$-measurable, $\mathscr{G}$ is a sub- $\sigma$-field of $\mathscr{F}_{(-\infty, a]}$. Since $H_{\Gamma}$ is a separable Hilbert space, $\mathscr{G}$ is countably generated. Hence there exists a sequence of finite $\sigma$-fields, $\mathscr{G}_{n} \uparrow \mathscr{G}$. Let $W_{\mathscr{G}}^{*}, W_{\mathscr{G}_{n}}^{*}, n=1,2,3, \ldots$, be the Hilbert subspaces of $W_{a}^{*}$ consisting of those $f \in W_{a}^{*}$ such that for each $\phi \in \mathscr{D}$, $(f, \phi)$ is $\mathscr{G}$-measurable, $\mathscr{G}_{n}$-measurable, respectively. Note that for each $n, W_{\mathscr{G}_{n}}^{*} \subset \hat{W}_{a}$ and that $W_{\mathscr{G}_{n}}^{*} \uparrow W_{\mathscr{G}}^{*}$. Therefore if $E_{\mathscr{G}_{n}}(f)$ is the projection of $f$ on $W_{\mathscr{G}_{n}}^{*}$, then $f=\lim _{n \rightarrow \infty} E_{\mathscr{G}_{n}}(f)$ in $W_{a}^{*}$ (Doob, [1, IV, Theorem 7.4]).

Hence the isometry given by equation (4.1) can be extended to an isometry from $W_{a}^{*}$ to $L^{2}(\Omega, \mathscr{F}, P)$.

Proposition 4.3. If $f \in W_{a}^{*}$, then $E\left(X(f) \mid \mathscr{F}_{(-\infty, a]}^{X}\right)=0$.

Proof. It suffices to show this for $f(t, \omega)=\chi_{A_{i}}(\omega) \bar{f}(t), A_{i} \in \mathscr{F}_{(-\infty, a]}^{X}, \bar{f}(t) \in H_{\Gamma}$. But then

$$
\begin{aligned}
E\left(X(f) \mid \mathscr{F}_{(-\infty, a]}^{X}\right) & =E\left(\chi_{A_{t}}(\omega) X(\bar{f})(\omega) \mid \mathscr{F}_{(-\infty, a]}^{X}\right) \\
& =\chi_{A_{i}}(\cdot) E\left(X(\bar{f}) \mid \mathscr{F}_{(-\infty, a]}^{X}\right) \\
& =\chi_{A_{i}}(\cdot) E(X(\bar{f})) \text { since } X(\bar{f}) \text { is } \mathscr{F}_{[a, \infty)}^{X} \text {-measurable } \\
& =0 \text { by Proposition 3.4. }
\end{aligned}
$$

Proposition 4.4 There exists a linear mapping $X(\cdot)$ from $W^{* 0} \cap W^{N}$ to $L^{2}(\Omega, \mathscr{F}, P)$ which preserves inner products and which agrees with that given by equation 4.1 on $W_{a}^{*}, a \in R^{1}$.

Proof. Consider $f \in W^{* 0} \cap W^{N}$ and let $a_{0}, \ldots, a_{m}$ be a partition of Spt $(f)$. Let

$$
\begin{aligned}
& f_{0}=\text { Projection of } f \text { on } W_{a_{0}}^{*}, \\
& f_{1}=\text { Projection of } f-f_{0} \text { on } W_{a_{1}}^{*}, \\
& f_{m}=\text { Projection of }\left(f-f_{1} \cdots-f_{m-1}\right) \text { on } W_{a_{m}}^{*} .
\end{aligned}
$$

Then set

$$
f_{\left\{a_{i}\right\}}(t, \omega) \equiv \sum_{i=0}^{m} f_{i}(t, \omega)
$$

We now prove that the mapping $\sum_{i=0}^{m} f_{i} \leadsto \sum_{i=0}^{m} X\left(f_{i}\right)$ preserves inner products, that is

$$
E\left(\sum_{i=0}^{m} X\left(f_{i}\right) \cdot \sum_{j=0}^{m} X\left(g_{j}\right)\right)=\left\langle\sum_{i=0}^{m} f_{i}, \sum_{j=0}^{m} g_{j}\right\rangle_{\Gamma} .
$$

To prove this, it suffices to show that if $f=\chi_{A} \bar{f}, g=\chi_{B} \bar{g}, \bar{f}$ and $\bar{g} \in H_{\Gamma}$ with appropriate supports, $A \in \mathscr{F}_{(-\infty, a]}^{X}, B \in \mathscr{F}_{(-\infty, b]}^{X}, a<b, \bar{f} \perp \bar{g}$ in $H_{\Gamma}$, then $E(X(f) \cdot X(g))=0$. Since 
$\mathscr{F}_{(-\infty, b-1 / n]}^{X} \uparrow \mathscr{F}_{(-\infty, b]}^{X}$ as $n \uparrow \infty$, there exist $B_{n}, n=1,2,3, \ldots$, such that $B_{n} \in$ $\mathscr{F}_{(-\infty, b-1 / n]}^{X}$ and $\chi_{B_{n}}(\cdot) \rightarrow \chi_{B}(\cdot)$ a.s. Then $\chi_{B_{n}}(\cdot) \bar{g} \rightarrow \chi_{B} \bar{g}$ in $\langle\cdot, \cdot\rangle_{\Gamma}$ and $\chi_{B_{n}} X(\bar{g}) \rightarrow$ $\chi_{B} X(\bar{g})$ in $L^{2}(\Omega, \mathscr{F}, P)$. Hence it suffices to show that $E\left(\left(\chi_{A} X(\bar{f})\right) \cdot\left(\chi_{B_{n}} X(\bar{g})\right)=0\right.$ for each $n$.

Any $\bar{f}$ with support in $[a, \infty)$ can be decomposed as $\bar{f}=\bar{f}_{1}+\bar{f}_{2}$ with $\operatorname{Spt}\left(\bar{f}_{1}\right) \subset[a, b)$ and Spt $\left(\bar{f}_{2}\right) \subset[b-1 / n, \infty), \bar{f}_{1}$ and $\bar{f}_{2} \in H_{0}^{2, N}$. Then

But

$$
\begin{aligned}
E\left(\left(\chi_{A} X(\bar{f})\right) \cdot\left(\chi_{B_{n}} X(\bar{g})\right)=\right. & E\left(\left(\chi_{A} X\left(\bar{f}_{1}\right)\right)\left(\chi_{B_{n}} X(\bar{g})\right)\right) \\
& +E\left(\left(\chi_{A} X\left(\bar{f}_{2}\right)\right) \cdot\left(\chi_{B_{n}} X(\bar{g})\right)\right) .
\end{aligned}
$$

$$
\begin{aligned}
E\left(\left(\chi_{A} X\left(\bar{f}_{1}\right)\right) \cdot\left(\chi_{B_{n}} X(\bar{g})\right)\right) & =E\left(E\left(\left(\chi_{A} X\left(\bar{f}_{1}\right)\right)\left(\chi_{B_{n}} X(\bar{g})\right) \mid \mathscr{F}_{(-\infty, b]}\right)\right) \\
& =E\left(\chi_{A} X\left(\bar{f}_{1}\right) \chi_{B_{n}} E\left(X(\bar{g}) \mid \mathscr{F}_{(-\infty, b]}\right)\right) \\
& =0 \text { by Proposition 4.3. }
\end{aligned}
$$

We have

$$
\begin{aligned}
\left\langle\chi_{A} \bar{f}_{1}, \chi_{B_{n}} \bar{g}\right\rangle_{\Gamma} & =E\left[\chi_{A}(\omega) \chi_{B_{n}}(\omega) \int_{-\infty}^{\infty} \sum_{j, k=0}^{n} r_{j k}(x) \bar{f}_{1}^{(j)}(x) \bar{g}^{(k)}(x) d x\right] \\
& =0 \text { since } \operatorname{Spt}\left(\bar{f}_{1}\right) \cap \operatorname{Spt}(\bar{g})=\varnothing
\end{aligned}
$$

and therefore $\left\langle\chi_{A} \bar{f}_{2}, \chi_{B_{n}} \bar{g}\right\rangle_{\Gamma}=0$. But by Propositions 4.1 and 4.2,

$$
E\left(\left(\chi_{A} X\left(\bar{f}_{2}\right)\right) \cdot\left(\chi_{B_{n}} X(\bar{g})\right)\right)=\left\langle\chi_{A} \bar{f}_{2}, \chi_{B_{n}} \bar{g}\right\rangle_{\Gamma}=0,
$$

since both $\chi_{A} \bar{f}_{2}$ and $\chi_{B_{n}} \bar{g}$ are in $W_{b-1 / n}^{*}$.

To complete the proof we must show that

$$
\lim _{\mathscr{P}} f_{\left\{a_{i}\right\}}=f \text { in } W^{* 0}
$$

where $\mathscr{P}$ is the directed set of partitions.

We first show that the $C^{\infty}$-functions in $W^{* 0} \cap W^{N}$ are actually dense in $W^{* 0}$. Consider $\rho_{\varepsilon}(x) \equiv(1 / \varepsilon) \rho(x / \varepsilon-1)$ where

$$
\begin{aligned}
\rho(x) & =a \exp \left(\frac{-1}{1-|x|^{2}}\right) & & \text { for }|x|<1, \\
& =0 & & \text { for }|x| \geqq 1 .
\end{aligned}
$$

where

$$
a^{-1}=\int_{|x|<1} \exp \left(\frac{-1}{1-|x|^{2}}\right) d x \text {. }
$$

Then for any $f \in W^{* 0},\left(f * \rho_{\varepsilon}\right)(\cdot, \omega) \rightarrow f(\cdot, \omega)$ in $H_{0}^{2, N}$ for almost every $\omega$ as $\varepsilon \downarrow 0$ (cf. Treves [20, p. 328]). In addition

$$
\left\|f * \rho_{\varepsilon}-f\right\|_{2, N}(\omega) \leqq\|f\|_{2, N}(\omega)\left[1+\|\rho\|_{L^{1}}\right] \text { for a.e. } \omega .
$$

Hence by the bounded convergence theorem, $f * \rho_{\varepsilon} \rightarrow f$ in $W^{*}$. To complete the proof note that for $\varepsilon>0, f * \rho_{\varepsilon} \in W^{0 *} \cap W^{N}$ and for almost every $\omega, f * \rho_{\varepsilon}(\cdot, \omega)$ is $C^{\infty}$. Hence it suffices to verify equation (4.3) for an a.s. $C^{\infty}$-function in $W^{0 *} \cap W^{N}$. 
If $h>0, f^{h}(t, \omega) \equiv f(t-h, \omega)$, then for each $t, f^{h}(t, \omega)$ is $\mathscr{F}_{(-\infty, t-h]^{X}}^{X}$ measurable. Hence it is clear that $\lim _{\mathscr{P}} f_{\left\{a_{i}\right\}}^{h}=f^{h}$ in $W^{0 *}$. On the other hand, if $f$ is a.s. $C^{\infty}$, then $\left\|f-f^{h}\right\|_{\Gamma}^{2} \rightarrow 0$ a.s. as $h \rightarrow 0$ [1, p. 441].

Since $\left\|f-f^{h}\right\|_{\Gamma}^{2} \leqq \alpha\|f\|_{2, N}^{2}$ a.s., the bounded convergence theorem implies that $f^{h} \rightarrow f$ in $W^{*}$, as $h \rightarrow 0$. Hence if $f \in W^{0 *}$ is a.s. $C^{\infty}$,

$$
\left\|f-f_{\left\{a_{i}\right\}}\right\|_{\Gamma} \leqq\left\|f-f^{h}\right\|_{\Gamma}+\left\|f^{h}-f_{\left\{a_{i}\right\}}^{h}\right\|_{\Gamma}+\left\|f_{\left\{a_{i}\right\}}^{h}-f_{\left\{a_{i}\right\}}\right\|_{\Gamma} .
$$

But the right-hand side of (4.4) can be made less than $\varepsilon>0$ by first choosing $h$ so that $\left\|f-f^{h}\right\|_{\Gamma}<\varepsilon / 3$ and then choosing $\left\{a_{i}\right\}$ so that $\left\|f^{h} f_{\left\{a_{i}\right\}}^{h}\right\|_{\Gamma}<\varepsilon / 3$. The proof is then complete.

REMARK 4.1. If we had not assumed that $\Gamma(\cdot, \cdot)$ were of the form of equation 3.1 but rather had tried to deal with the general form of Theorem 2.4, in which the measure $\mu$ need not be absolutely continuous with respect to Lebesgue measure, then the proof of Proposition 4.4 would not go through as given. This corresponds to the situation which has arisen in the extension of the Itô integral to the stochastic integrals with respect to square integrable martingales. This has been carefully studied by $P$. Courrège and $P$. A. Meyer [13] and requires the investigation of rather delicate measurability considerations.

Propositions 4.1, 4.2 and 4.4 can be summarized in the following theorem.

THEOREM 4.1. There exists a linear isometry $X(\cdot)$ from $W^{*}$ to $L^{2}(\Omega, \mathscr{F}, P)$ such that if $f, g \in W^{N}$

$$
E(X(f) X(g))=\langle f(\cdot, \cdot), g(\cdot, \cdot)\rangle_{\Gamma} .
$$

Proof. This follows immediately from the proof of the above proposition since $W^{0 *} \cap W^{N}$ is dense in $W^{*}$.

Proposition 4.5. If $f \in W^{*}$ and $\operatorname{Spt}(f) \subset[a, \infty)$, then $E\left(X(f) \mid \mathscr{F}_{(-\infty, a]}^{X}\right)=0$.

Proof. It suffices to show this for a sum of the type (4.2) where $a_{0}=a$. Then

$$
\begin{aligned}
E\left(X(f) \mid \mathscr{F}_{(-\infty, a]}^{X}\right) & =\sum_{i} E\left(X\left(f_{i}\right) \mid \mathscr{F}_{(-\infty, a]}^{X}\right) \\
& =\sum_{i} E\left(E\left(X\left(f_{i}\right) \mid \mathscr{F}_{\left(-\infty, a_{i}\right]}^{X}\right) \mid \mathscr{F}_{(-\infty, a]}^{X}\right) \\
& =0 \text { by Proposition 4.3. }
\end{aligned}
$$

If $\Phi: \mathscr{D} \rightarrow W^{*}$ is a continuous linear mapping, then so is the composition

$$
\mathscr{D} \stackrel{\Phi}{\longrightarrow} W^{*} \stackrel{X}{\longrightarrow} L^{2}(\Omega, \mathscr{F}, P) .
$$

The composition then yields a generalized stochastic process which has a modification which is a second order random distribution with correlation functional

$$
\Gamma(\phi, \psi)=E\left[\int_{-\infty}^{\infty} \sum_{j, k=0}^{N} r_{j k}(x) \Phi(\phi)^{(j)}(x) \Phi(\psi)^{(k)}(x) d x\right] .
$$


We now apply this to obtain the $P$-products of $f \in W^{N}$ and $X$. For fixed $f \in W^{N}$ consider the linear mapping from $\mathscr{D}$ to $W^{*}$ defined by $\phi \leadsto \phi f$.

Proposition 4.6. If $f \in W^{N}$, then the mapping $\phi \leadsto f \phi$ is continuous.

Proof. The proof is essentially the same as that of Proposition 3.3.

Therefore the $P$-transformation of $X$ by the mapping $\phi \leadsto f \phi$ yields a random distribution which is called the $P$-product of $f$ and $X$. If $f \in \mathscr{D}$ a.s., then for almost every $\omega, X f(\omega)$ is the product of $f(\omega)$ with the distribution $X(\omega)$ in the usual sense of the theory of distributions. In addition we then have $(X f)(\phi)$ is the stochastic integral of $\phi$ with respect to $X f$. We denote by $\int^{t} X f$ the primitive of the random distribution $X f$; that is, if $\phi \in \mathscr{D}$, then

$$
\left(\int^{t} X f\right)(\dot{\phi})=-(X f)(\phi), \text { where } \dot{\phi}(t)=d \phi(t) / d t .
$$

If we fix $\phi_{0} \in \mathscr{D}$ which is not of the form $\dot{\psi}$ for $\psi \in \mathscr{D}$, then we can allow $\left(\int^{t} X f\right)\left(\phi_{0}\right)$ to be an arbitrary random variable with finite second moment.

Note 4.1. A note on the calculus of $P$-transformations.

As we have seen above the definition of a stochastic integral with respect to a generalized process with independent values is an extension of the Itô calculus. From such a point of view it is desirable to establish the rules satisfied by the calculus. However, in this section we settle for demonstrating that the rule for the differentiation of a product is satisfied (which is also the basis for integration by parts).

Proposition 4.7. If $f \in W^{N+1}$, then

$$
d(X f) / d t=X d f / d t+f d X / d t .
$$

Proof. Let $f_{n} \equiv f * \rho_{1 / n}$ where $\rho_{\varepsilon}$ is as defined in the proof of Proposition 4.4. Then $f_{n} \rightarrow f$ in $W^{N+1}$ and $\dot{f}_{n} \rightarrow \dot{f}$ in $W^{N}$. Now by a theorem of Schwartz [16, Chapter IV, Theorem 4],

$$
d\left(X f_{n}\right) / d t=X d f_{n} / d t+f_{n} d X / d t \text { for each } n .
$$

But for any $\phi \in \mathscr{D},\left(X d f_{n} / d t\right)(\phi) \rightarrow(X d f / d t)(\phi)$ in $L^{2}(\Omega, \mathscr{F}, P),\left(f_{n} d X / d t\right)(\phi)$ $\rightarrow(f d X / d t)(\phi)$ in $L^{2}(\Omega, \mathscr{F}, P)$, and $\left(X f_{n}\right)(\phi) \rightarrow(X f)(\phi)$ in $L^{2}(\Omega, \mathscr{F}, P)$.

Therefore, by Theorem 2.3 , there exists a subsequence $\left\{n_{k}\right\}$ such that

$$
\begin{aligned}
& X d f_{n_{k}} / d t \rightarrow X d f / d t \text { in } \mathscr{D}^{\prime} \quad \text { a.s. } \\
& f_{n_{k}} d X / d t \rightarrow f d X / d t \text { in } \mathscr{D}^{\prime} \quad \text { a.s. } \\
& \left(X f_{n_{k}}\right) \rightarrow X f \text { in } \mathscr{D}^{\prime} \quad \text { a.s. }
\end{aligned}
$$

and therefore

$$
d\left(X f_{n_{k}}\right) / d t \rightarrow d(X f) / d t \text { in } \mathscr{D}^{\prime} \text { a.s. }
$$

Equation (4.5) then follows from equations (4.6) and (4.7). 
COROLlaRY. If $f \in W^{N+1}$ has compact support, then $X(\dot{f})=-\dot{X}(f)$.

Proof. Let $\phi \in \mathscr{D}$ such that $\phi(x)=1$ for $x \in \operatorname{Spt}(f)$.

Then by 4.5

$$
-(X f)(\dot{\phi}) \equiv-X(f \dot{\phi})=(X(d f / d t))(\phi)+((d X / d t) f)(\phi) .
$$

But $f \dot{\phi}=0$, and therefore

$$
(X(d f / d t))(\phi)=-((d X / d t) f)(\phi) .
$$

Therefore,

$$
X((d f / d t) \phi)=-(d X / d t)(f \phi) .
$$

But $(d f / d t) \phi=d f / d t$ and $f \phi=f$ and therefore $X(d f / d t)=-(d X / d t)(f)$.

REMARK 4.2. It should be noted that in general the stochastic calculus is not identical to ordinary calculus. For example, putting K. Itô's formula for stochastic differentials [8] into our setting, one can show that

$$
d e^{B(t)} / d t=\frac{1}{2} e^{B(t)}+e^{B(t)} d B / d t \text { a.s. }
$$

4.8. Connection with the Itô integral. We now show that the stochastic integral defined above agrees with the Itô stochastic integral when $X=\dot{B}$. In this case $W^{*}=V^{M}$. Hence it suffices to show that the two integrals agree on $(t, \omega)$-step functions.

Let $f(t, \omega)=\sum_{j=0}^{n} \chi_{\left[a_{j}, a_{j+1}\right)}(t) f_{j}(\omega)$ where each $f_{j}(\cdot)$ is $\mathscr{F}_{\left(-\infty, a_{j}\right]}$-measurable. Then since the functions $\chi_{\left[a_{j}, a_{j+1}\right)} f_{j}(\cdot)$ are orthogonal in $W^{*}$, it suffices to show that the integrals agree for $\chi_{\left[a_{j}, a_{j+1}\right)}(t) f_{j}(\omega)$. But $\chi_{\left[a_{j}, a_{j+1}\right)}(t) f_{j}(\omega) \in W_{a_{j}}^{*}$ and $f_{j}(\omega)$ can be approximated in $L^{2}(\Omega, \mathscr{F}, P)$ by functions of the form $\sum_{i=1}^{n} b_{i} \chi_{B_{i}}(\omega)$ where the $B_{i} \in \mathscr{F}_{\left(-\infty, a_{j}\right]}$ and $B_{i} \cap B_{k}=\varnothing$ if $i \neq k$.

Hence it suffices to show that the integrals agree for functions of the form

$$
b_{i} \chi_{B_{i}}(\omega) \chi_{\left[a_{j}, a_{j+1}\right)}(t) \in \hat{W}_{a_{j}} .
$$

But then

$$
\dot{B}\left(b_{i} \chi_{B_{i}}(\omega) \chi_{\left[a_{j}, a_{j+1}\right)}\right)=b_{i} \chi_{B_{i}}(\omega) \dot{B}\left(\chi_{\left[a_{j}, a_{j+1}\right)}\right) .
$$

But it is shown in Example 3.1 that

$$
\dot{B}\left(\chi_{\left[a_{j}, a_{j+1}\right)}(\cdot)\right)=\int_{-\infty}^{\infty} \chi_{\left[a_{j}, a_{j+1}\right)}(t) d B(t) .
$$

\section{Stochastic equations.}

5.1. Introduction. K. Itô and M. Nisio [9] have studied stochastic integral equations of the form

$$
X(t)=X(0)+\int_{0}^{t} a(s, X) d s+\int_{0}^{t} b(s, X) d B(s)
$$


with past condition $X(t)=X-(t)$ for $t \leqq 0$ where $a(s, X)$ and $b(s, X)$ are progressively measurable. In general, the stochastic integral equations of Itô deal with the case in which the processes integrated with respect to are either Brownian white noise or random measures.

On the other hand the eoncept of stochastic differential equations can be extended to any equation of the form

$$
\sum_{i=1}^{m} a_{i}(t) \frac{d^{i} Y}{d t^{i}}=X
$$

where $X$ is a generalized process and the $a_{i}(t)$ are $C^{\infty}$-functions.

THEOREM 5.1. Equation (5.1) has a solution which is a generalized process. Any two solutions differ by a solution of the homogeneous equations.

Proof. For each $\omega \in \Omega$, apply Theorem 2.15 to the equation

$$
\sum_{i=1}^{m} a_{i}(t) \frac{d^{i}}{d t^{i}} Y(\omega)=X(\omega) .
$$

J. L. Strand [19] has studied the above equation and obtained conditions under which the solutions have $p$ th moments. In a similar way we could consider a linear Volterra integral equation with an analytic kernel, for example,

$$
Y(t)+\int_{0}^{t} h(s, t) Y(s) d s=X(t), \quad X \text { a generalized process. }
$$

5.2. Generalized Itô equations. In this section we consider the family of stochastic differential equations of the form

$$
\frac{d Y_{i}}{d t}=a_{i}(\bar{Y})+\sum_{k=1}^{L} b_{i}^{k}(\bar{Y}) X_{k}, \quad i=1, \ldots, m,
$$

where $\left\{X_{k}\right\}$ are a family of independent generalized processes with independent values, and $a_{i}(\bar{Y})$ and $b_{i}^{k}(\bar{Y})$ are progressively measurable mappings from ${ }^{m} \mathscr{D}^{\prime}$ into a function space $S$.

A mapping $\psi$ from ${ }^{m} \mathscr{D}^{\prime}$ (the space of continuous linear functionals of $\mathscr{D}$ into $R^{m}$ ) into a space, $S$, of real valued functions on $R^{1}$ is progressively measurable if the mapping $\psi \circ E_{t}:\left({ }^{m} \mathscr{D}^{\prime}\right) \times(-\infty, t] \rightarrow R^{1}$ is measurable with respect to $\mathscr{B}\left({ }^{m} \mathscr{D}^{\prime},(-\infty, t]\right) \times \mathscr{B}\left(R^{1} ;(-\infty, t]\right)$ where $E_{t}$ is the "evaluation at $t$ " mapping of $S$ into $R^{1}$.

If $S$ is a space of distributions a similar definition is used with $E_{t}$ replaced by $E \phi$, the "evaluation at $\phi$ " mapping of $S$ into $R^{1}$; that is, $\psi \circ E \phi:{ }^{m} \mathscr{D}^{\prime} \times \mathscr{D}(-\infty, t]$ $\rightarrow R^{1}$ is measurable with respect to $\mathscr{B}\left({ }^{m} \mathscr{D}^{\prime},(-\infty, t]\right) \times \mathscr{B}(\mathscr{D}(-\infty, t])$.

By a solution to equation (5.2) we mean a $R^{m}$-valued generalized process $\bar{Y}$ defined over $(\Omega, \mathscr{F}, P)$ for which $b_{i}^{k}(\bar{Y})$ and $a_{i}(\bar{Y})$ are defined for each $i$ and $k$ and such that for every $\phi$ and $1 \leqq i \leqq m$, both sides of (5.2) evaluated at $\phi$ are equal 
almost surely. Note that this is all that can be expected since the right-hand side is defined uniquely only up to modifications. We first study the case $m=1, L=1$ and then modify this to study the general case.

TheOREM 5.2. Let $X$ be a second order generalized process on $(\Omega, \mathscr{F}, P)$ with independent values and whose correlation functional $\Gamma$ satisfies $\gamma=\sum_{j, k}\left\|r_{j, k}\right\|_{\infty}<\infty$. Assume that $b: \mathscr{D}^{\prime} \rightarrow H_{0}^{2, N}$ is progressively measurable and continuous and that $a: \mathscr{D}^{\prime} \rightarrow \mathscr{D}^{\prime}$ is continuous and progressively measurable. Assume that $\operatorname{Spt}(X) \subset[0, \infty)$ and that if Spt $(\phi) \subset(-\infty, 0]$ then $a(\cdot)(\phi)$ is zero. Let $V$ be an absorbing subset of $\mathscr{D}$ on which $\|\phi\|_{\infty, N}$ is bounded. For $s \in R^{1}$, let

$$
\|Y\|_{s}=\sup _{\phi \in V ; \operatorname{spt}(\phi) \subset(-\infty, s]} E(Y(\phi))^{2} .
$$

Assume that $\|a(0)+b(0) X\|_{s}$ is finite valued. Assume that for any progressively measurable second order generalized processes $Y_{1}, Y_{2}$,

$$
\left\|b\left(Y_{1}\right)-b\left(Y_{2}\right)\right\|_{2, N, s}^{2} \leqq K_{1} \int_{-\infty}^{s}\left\|Y_{1}-Y_{2}\right\|_{s}^{2} d s
$$

and

$$
\left\|a\left(Y_{1}\right)-a\left(Y_{2}\right)\right\|_{s}^{2} \leqq K_{2} \int_{-\infty}^{s}\left\|Y_{1}-Y_{2}\right\|_{s}^{2} d s
$$

Then the stochastic equation

$$
Y=a(Y)+b(Y) X
$$

has a unique solution.

Proof. Let $Y_{0} \equiv 0$ and (5.6) $Y_{n+1}=a\left(Y_{n}\right)+b\left(Y_{n}\right) X$. By (5.3) and (5.4) it follows that $Y_{1}$ is a second order $\mathscr{F}^{X}$-progressively measurable generalized process. If $\phi \in$ $V$ and $\operatorname{Spt}(\phi) \subset(-\infty, s]$, then by $(5.6)$

$$
\left(Y_{n+1}-Y_{n}\right)(\phi)=\left(a\left(Y_{n}\right)-a\left(Y_{n-1}\right)\right)(\phi)+\left(b\left(Y_{n}\right)-b\left(Y_{n-1}\right)\right) X(\phi) .
$$

Therefore

$$
\begin{aligned}
E\left[\left(Y_{n+1}-Y_{n}\right)(\phi)\right]^{2} & \leqq 2 E\left[\left(a\left(Y_{n}\right)-a\left(Y_{n-1}\right)\right)(\phi)\right]^{2}+2 E\left[\left(b\left(Y_{n}\right)-b\left(Y_{n-1}\right)\right) X(\phi)\right]^{2} \\
& \leqq 2 E\left[\left(a\left(Y_{n}\right)-a\left(Y_{n-1}\right)\right)(\phi)\right]^{2}+2 \gamma\|\phi\|_{\infty, N}^{2}\left\|b\left(Y_{n}\right)-b\left(Y_{n-1}\right)\right\|_{2, N, s}^{2} \\
& \leqq 2\left\|a\left(Y_{n}\right)-a\left(Y_{n-1}\right)\right\|_{s}^{2}+2 \gamma\|\phi\|_{\infty, N}^{2}\left\|b\left(Y_{n}\right)-b\left(Y_{n-1}\right)\right\|_{2, N, s}^{2} \\
& \leqq 2 K_{2} \int_{-\infty}^{s}\left\|Y_{n}-Y_{n-1}\right\|_{s}^{2} d s+2 \gamma\|\phi\|_{\infty, N}^{2} K_{1} \int_{-\infty}^{s}\left\|Y_{n}-Y_{n-1}\right\|_{s}^{2} d s \\
& \leqq K_{3} \int_{-\infty}^{s}\left\|Y_{n}-Y_{n-1}\right\|_{s}^{2} d s \text { since }\|\phi\|_{\infty, N} \text { is bounded on } V .
\end{aligned}
$$

Therefore

$$
\left\|Y_{n+1}-Y_{n}\right\|_{s}^{2} \leqq K_{3} \int_{0}^{s}\left\|Y_{n}-Y_{n-1}\right\|_{s}^{2} d s
$$


Hence by induction

$$
\begin{aligned}
\left\|Y_{n+1}-Y_{n}\right\|_{s}^{2} & \leqq K_{3}^{n} \int_{0}^{s} \cdots \int_{0}^{t_{n-1}}\left\|Y_{1}-Y_{0}\right\|_{t_{n}}^{2} d t_{n} d t_{n-1} \cdots d t_{2} \\
& \leqq \frac{K_{3}^{n} s^{n}}{n !}\left\|Y_{1}-Y_{0}\right\|_{s}^{2} .
\end{aligned}
$$

For each $\phi \in \mathscr{D}$ there is an $\alpha \in(0, \infty)$ such that $\alpha \phi \in V$ since $V$ is absorbing. Then,

$$
\begin{aligned}
E\left(\left(Y_{n+1}-Y_{n}\right) \phi\right)^{2} & =\frac{1}{\alpha^{2}} 2 E\left(\left(Y_{n+1}-Y_{n}\right) \alpha \phi\right)^{2} \\
& \leqq \frac{1}{\alpha^{2}}\left\|Y_{n+1}-Y_{n}\right\|_{s}^{2} \leqq \frac{K_{3}^{n} s^{n}}{\alpha^{2} n !}\left\|Y_{1}-Y_{0}\right\|_{s}^{2}
\end{aligned}
$$

Hence

$$
\begin{aligned}
{\left[E\left(\left(Y_{m}-Y_{n}\right) \phi\right)^{2}\right]^{1 / 2} } & =\left[E\left(\sum_{r=m}^{n-1}\left(Y_{r+1}-Y_{r}\right) \phi\right)^{2}\right]^{1 / 2} \\
& \leqq \sum_{r=m}^{n-1}\left(E\left(\left(Y_{r+1}-Y_{r}\right) \phi\right)^{2}\right)^{1 / 2} \\
& \leqq \sum_{r=m}^{n-1}\left(\frac{K_{3}^{r} s^{r}}{\alpha^{2} r !}\right)^{1 / 2} \cdot\left\|Y_{1}-Y_{0}\right\|_{s}
\end{aligned}
$$

Since the series $\sum_{n=1}^{\infty} a^{n / 2} /(n !)^{1 / 2}$ is convergent,

$$
E\left(\left(Y_{m}-Y_{n}\right) \phi\right)^{2} \rightarrow 0 \text { as } m, n \rightarrow \infty .
$$

Therefore Theorem 2.3 implies that there exists a subsequence $\left\{Y_{n_{k}}\right\}$ such that $Y_{n_{k}}$ converges almost surely to a generalized process $Y_{\infty}$ in the sense of convergence in $\mathscr{D}^{\prime}$. Since $a(\cdot)$ and $b(\cdot)$ are continuous, $a\left(Y_{n_{k}}\right) \rightarrow a\left(Y_{\infty}\right)$ in $\mathscr{D}^{\prime}$ a.s. and $b\left(Y_{n_{k}}\right)$ $\rightarrow b\left(Y_{\infty}\right)$ in $H_{0}^{2, N}$ a.s. Moreover (5.3) implies that $b\left(Y_{n_{k}}\right)$ is a Cauchy sequence with respect to $\|\cdot\|_{2, N, t}$ for each $t$ and therefore $b\left(Y_{n_{k}}\right) \rightarrow b\left(Y_{\infty}\right)$ with respect to $\|\cdot\|_{2, N, t}$ for each $t$. Hence for each $\phi \in \mathscr{D}, b\left(Y_{n_{k}}\right) X(\phi) \rightarrow b\left(Y_{\infty}\right) X(\phi)$ in $L^{2}(\Omega, \mathscr{F}, P)$. Equation (5.4) implies that for each $\phi, a\left(Y_{n_{k}}\right)(\phi) \rightarrow a\left(Y_{\infty}\right)(\phi)$ in $L^{2}(\Omega, \mathscr{F}, P)$ and we have shown above that $Y_{n_{k}}(\phi) \rightarrow Y_{\infty}(\phi)$ in $L^{2}(\Omega, \mathscr{F}, P)$.

Therefore

and

$$
E\left(\left(Y_{\infty}-a\left(Y_{\infty}\right)-b\left(Y_{\infty}\right) X\right)(\phi)\right)^{2}=0
$$

$$
Y_{\infty}(\phi)=a\left(Y_{\infty}\right)(\phi)+\left(Y_{\infty}\right) X(\phi) \text { a.s. }
$$

Hence there is a modification of $b\left(Y_{\infty}\right) X$ such that $Y_{\infty}=a\left(Y_{\infty}\right)+b\left(Y_{\infty}\right) X$ a.s.

We next prove the uniqueness (up to modification).

Let $Y_{\infty}^{1}$ and $Y_{\infty}^{2}$ be two solutions of (5.5). Then as above if $\phi \in V$, Spt $(\phi) \subset(-\infty, s]$, then

$$
E\left[\left(Y_{\infty}^{1}-Y_{\infty}^{2}\right)(\phi)\right]^{2} \leqq \frac{K_{3}^{n} s^{n}}{n !}\left\|Y_{\infty}^{1}-Y_{\infty}^{2}\right\|_{s}^{2}
$$


Therefore,

$$
\left\|Y_{\infty}^{1}-Y_{\infty}^{2}\right\|_{s}^{2} \leqq \frac{K_{3}^{n} s^{n}}{n !}\left\|Y_{\infty}^{1}-Y_{\infty}^{2}\right\|_{s}^{2}
$$

Since for sufficiently large $n, K_{3}^{n} s^{n} / n !<1$,

$$
\left\|Y_{\infty}^{1}-Y_{\infty}^{2}\right\|_{s}^{2}=0 \quad \text { and hence for each } \phi \in \mathscr{D}, \quad Y_{\infty}^{1}(\phi)=Y_{\infty}^{2}(\phi) \text { a.s. }
$$

COROLLARY. Under the above hypotheses the initial value problem

$$
\dot{Y}=a(\dot{Y})+b(\dot{Y}) X, \quad Y(\phi)=0 \text { if Spt }(\phi) \subset(-\infty, 0]
$$

has a unique solution.

EXAMPLE 5.1. The following is a very simple example of a mapping $b(\cdot)$ which satisfies the hypotheses of Theorem 5.2.

Let $b(Y) \equiv \phi_{0} * Y$ for some $\phi_{0} \in \mathscr{D}$ with support in $[0, \infty)$. It is easy to verify that $b(\cdot)$ is progressively measurable and continuous from $\mathscr{D}^{\prime}$ to $\mathscr{D}^{\prime}$. Then

$$
\begin{aligned}
\left\|b\left(Y_{1}\right)-b\left(Y_{2}\right)\right\|_{2, N, s}^{2} & =E\left[\int_{-\infty}^{s} \sum_{j=0}^{N}\left(\left(b\left(Y_{1}\right)-b\left(Y_{2}\right)\right)^{(j)}(x)\right)^{2} d x\right] \\
& =E\left[\int_{-\infty}^{s} \sum_{j=0}^{N}\left(\left(\phi_{0}^{(j)} *\left(Y_{1}-Y_{2}\right)(x)\right)\right)^{2} d x\right] \\
& =\int_{-\infty}^{s} \sum_{j=0}^{N} E\left(\left(Y_{1}-Y_{2}\right)\left(\phi_{0}^{(j)}(x-\cdot)\right)\right)^{2} d x .
\end{aligned}
$$

Since Spt $\left(\phi_{0}^{(j)}(x-\cdot)\right) \subset(-\infty, x]$, then if $V$ is any absorbing subset of $\mathscr{D}$ containing the functions $\phi_{0}^{(j)}(t-\cdot), t \in R^{1}, 1 \leqq j \leqq N$, it follows that

$$
\left\|b\left(Y_{1}\right)-b\left(Y_{2}\right)\right\|_{2, N, s}^{2} \leqq K \int_{-\infty}^{s}\left\|Y_{1}-Y_{2}\right\|_{s}^{2} d s .
$$

THEOREM 5.3. Let $X_{k}, k=1, \ldots, L$ be independent second order generalized processes with zero means and independent values and correlation functionals $\Gamma_{k}$ which satisfy $\alpha=\sum_{i, j, k}\left\|r_{i, j}^{k}(\cdot)\right\|_{\infty}<\infty$.

Assume that $b_{i}^{k}:{ }^{M} \mathscr{D}^{\prime} \rightarrow H_{0}^{2, N_{k}}, \quad i=1, \ldots, M, k=1, \ldots, L$ are progressively measurable and continuous and that $a_{i}:{ }^{M} \mathscr{D}^{\prime} \rightarrow \mathscr{D}^{\prime}, i=1, \ldots, M$ are progressively measurable and continuous. Assume that Spt $\left(r_{i, j}^{k}\right) \subset[0, \infty), i, j \leqq N_{k}, k=1, \ldots, L$.

Assume that if Spt $(\phi) \subset(-\infty, 0]$, then $a_{i}(\cdot)(\phi)=0, i=1, \ldots, M$. Let

$$
\|\bar{Y}\|_{s}^{2} \equiv \sup \left\{E\left(|\bar{Y} \phi|^{2}\right): \phi \in V, \operatorname{Spt}(\phi) \subset(-\infty, s]\right\}
$$

and $V$ is an absorbing set on which $\|\phi\|_{\infty, N_{k}}, k=1, \ldots, L$ are bounded. Assume that $\left\|a_{i}(0)+\sum_{k=1}^{L} b_{i}^{k}(0) X_{k}\right\|_{s}$ are finite valued. Assume that for any pair $\bar{Y}_{1}, \bar{Y}_{2}$ of $\mathscr{F}^{X_{1}}, \ldots, x_{L}$ progressively measurable second order $R^{M}$-valued processes,

$$
\begin{array}{r}
\left\|b_{i}^{k}\left(\bar{Y}_{1}\right)-b_{i}^{k}\left(\bar{Y}_{2}\right)\right\|_{2, N_{k}, s}^{2} \leqq K_{i k}^{1} \int_{-\infty}^{s}\left\|\bar{Y}_{1}-\bar{Y}_{2}\right\|_{s}^{2} d s, \\
\qquad i=1, \ldots, M ; k=1, \ldots, L,
\end{array}
$$


and

$$
\left\|a_{i}\left(\bar{Y}_{1}\right)-a_{i}\left(\bar{Y}_{2}\right)\right\|_{s}^{2} \leqq K_{i}^{2} \int_{-\infty}^{s}\left\|\bar{Y}_{1}-\bar{Y}_{2}\right\|_{s}^{2} d s .
$$

Then the system of stochastic equations

$$
Y^{i}=a_{i}(\bar{Y})+\sum_{k=1}^{L} b_{i}^{k}(\bar{Y}) X_{k}, \quad i=1, \ldots, M,
$$

has a unique solution where $\bar{Y}=\left(Y^{1}, \ldots, Y^{M}\right)$.

Proof. Put $Y_{0}^{i}=0 ; i=1, \ldots, M$, and

$$
Y_{n+1}^{i}=a_{i}\left(\bar{Y}_{n}\right)+\sum_{k=1}^{L} b_{i}^{k}\left(\bar{Y}_{n}\right) X_{k}, \quad i=1, \ldots, M .
$$

If $\phi \in V$ and Spt $(\phi) \subset(-\infty, s]$, then

$$
\begin{aligned}
\left(Y_{n+1}^{i}-Y_{n}^{i}\right)(\phi)= & \left(a_{i}\left(\bar{Y}_{n}\right)-a_{i}\left(\bar{Y}_{n-1}\right)\right)(\phi) \\
& +\sum_{k=1}^{L}\left(b_{i}^{k}\left(\bar{Y}_{n}\right)-b_{i}^{k}\left(\bar{Y}_{n-1}\right)\right) X_{k}(\phi), \quad i=1, \ldots, M .
\end{aligned}
$$

Hence

$$
\begin{aligned}
E\left(\left(Y_{n+1}^{i}-Y_{n}^{i}\right)(\phi)\right)^{2} \leqq & (L+1)\left\{E\left(\left(a_{i}\left(\bar{Y}_{n}\right)-a_{i}\left(\bar{Y}_{n-1}\right)\right)(\phi)\right)^{2}\right. \\
& \left.+\sum_{k=1}^{L} E\left[\left(b_{i}^{k}\left(\bar{Y}_{n}\right)-b_{i}^{k}\left(\bar{Y}_{n-1}\right)\right) X(\phi)\right]^{2}\right\} \\
\leqq & (L+1)\left\{\left\|a_{i}\left(\bar{Y}_{n}\right)-a_{i}\left(\bar{Y}_{n-1}\right)\right\|_{s}^{2}\right. \\
& \left.+\alpha \sum_{k=1}^{L}\|\phi\|_{\infty, N_{k}}^{2}\left\|b_{i}^{k}\left(\bar{Y}_{n}\right)-b_{i}^{k}\left(\bar{Y}_{n-1}\right)\right\|_{2, N_{k}, s}^{2}\right\} \\
\leqq & (L+1)\left\{K_{i}^{1} \int_{-\infty}^{s}\left\|\bar{Y}_{n}-\bar{Y}_{n-1}\right\|_{s}^{2} d s\right. \\
& \left.+\alpha \sum_{k=1}^{L}\|\phi\|_{\infty, N_{k}}^{2} K_{i k}^{2} \int_{-\infty}^{s}\left\|\bar{Y}_{n}-\bar{Y}_{n-1}\right\|_{s}^{2} d s\right\} .
\end{aligned}
$$

Therefore if $\phi \in V$ and Spt $(\phi) \subset(-\infty, s]$, then

$$
E\left(\left|\left(\bar{Y}_{n+1}-\bar{Y}_{n}\right)(\phi)\right|^{2}\right) \leqq \tilde{K} \int_{-\infty}^{s}\left\|\bar{Y}_{n}-\bar{Y}_{n-1}\right\|_{s}^{2} d s
$$

where $\tilde{K}$ is a constant. Therefore,

$$
\left\|\bar{Y}_{n+1}-\bar{Y}_{n}\right\|_{s}^{2} \leqq \tilde{K} \int_{-\infty}^{s}\left\|\bar{Y}_{n}-\bar{Y}_{n-1}\right\|_{s}^{2} d s .
$$

We can then repeat the reasoning of Theorem 5.2 to show that $\bar{Y}_{n_{k}} \rightarrow \bar{Y}_{\infty}$ in ${ }^{M} \mathscr{D}^{\prime}$ almost surely for some subsequence $\left\{\bar{Y}_{n_{k}}\right\}$ and that $\bar{Y}_{\infty}$ satisfies the system of equations (5.8). 
In Theorems 5.2 and 5.3 existence proofs were obtained by working on $\mathscr{D}^{\prime}$ and using the basic properties of convergence of generalized processes. However, in many cases one must work in a Sobolev space $H^{2,-M}$ instead of all of $\mathscr{D}^{\prime}$. In such a case, it is more difficult to prove the existence of a solution. In the remainder of this section we will prove an illustrative existence theorem of this type, which is analogous to the usual Itô equations. We will require the following lemma.

Lemma 5.1. Assume that $X=d^{2} B / d t^{2}, b$ is $\mathscr{F}^{x}$ progressively measurable with Spt $(b) \subset[0, \infty)$ and for each $t,\|b\|_{2,1 ; t}^{2} \equiv E \int_{0}^{t}\left(b^{2}(t, \omega)+\dot{b}^{2}(t, \omega)\right) d t<\infty$.

Then for $\lambda>0$ there exists constants $K_{1}$ and $K_{2}$ such that

$$
P\left(\sup _{0 \leqq s \leqq t}\left|\int^{s} \int b(t, \omega) \frac{d^{2} B}{d t^{2}}\right| \geqq \lambda\right) \leqq \frac{K_{1}}{\lambda^{2}}\|b\|_{2,1 ; t}^{2}
$$

and

$$
E\left[\sup _{0 \leqq s \leqq t}\left(\iint b(t, \omega) \frac{d^{2} B}{d t^{2}}\right)^{2}\right] \leqq K_{2}\|b\|_{2,1 ; t} .
$$

Proof. For $t \geqq 0$, let

$$
\begin{aligned}
\phi^{t}(s) & \equiv(t-s) & & \text { if } 0 \leqq s \leqq t, \\
& \equiv(t+s) & & \text { if } 0 \geqq s \geqq-t, \\
& \equiv 0 & & \text { otherwise. }
\end{aligned}
$$

Then $b \cdot \phi^{t} \in W^{1}$ and by the corollary to Proposition 4.7,

$$
\left(b(\cdot)\left(d^{2} B / d t^{2}\right)\right)\left(\phi^{t}\right)=\int_{0}^{t} \int b(t, \omega) \frac{d^{2} B}{d t^{2}} d t \text { a.s. }
$$

Let $Y_{t} \equiv b\left(d^{2} B / d t^{2}\right) \phi^{t}$. For $s \leqq t$, let $Y_{t}=Y_{s}+W_{s}$. Then $E\left(Y_{t} \mid \mathscr{F}_{(-\infty, s]}^{X}\right)=Y_{s}$ $+E\left(W_{s} \mid \mathscr{F}_{(-\infty, s]}^{X}\right)$. Let

$$
Z_{s} \equiv E\left(Y_{t} \mid \mathscr{F}_{(-\infty, s]}^{X}\right) \quad \text { and } \quad \hat{W}_{s}=E\left(W_{s} \mid \mathscr{F}_{(-\infty, s]}^{X}\right) .
$$

Then $Y_{s}=Z_{s}-\hat{W}_{s}$. Therefore, $\sup _{s \leqq t} Y_{s}^{2} \leqq 2\left(\sup _{s \leqq t} Z_{s}^{2}+\sup _{s \leqq t} \hat{W}_{s}^{2}\right)$.

Since $Z_{s}$ is a martingale, $Z_{s}^{2}$ is a submartingale and therefore by the submartingale inequality (Doob [1, Chapter VII, Theorem 3.2]),

where $K_{1}^{1}$ is a constant. Now

$$
\begin{aligned}
P\left(\sup _{s \leqq t} Z_{s}^{2} \geqq \lambda\right) & \leqq(1 / \lambda) E\left(Z_{t}^{2}\right)=(1 / \lambda) E\left(Y_{t}^{2}\right) \\
& =(1 / \lambda) E\left[\left(b d^{2} B / d t^{2}\right)\left(\phi^{t}\right)\right]^{2} \\
& =(1 / \lambda) E\left[\int_{0}^{t}\left(\left(\dot{b} \phi^{t}\right)^{2}(s)+\left(b \dot{\phi}^{t}\right)^{2}(s)\right) d s\right] \\
& =\left(K_{1}^{1} / \lambda\right) E\left[\int_{0}^{t}\left(\dot{b}^{2}(s)+b^{2}(s)\right) d s\right]
\end{aligned}
$$

$$
W_{s}=Y_{t}-Y_{s}=b\left(d^{2} B / d t^{2}\right)\left(\psi^{s}\right)
$$


where

$$
\begin{aligned}
\psi^{s}(u) & =(t-s) & & \text { for }-s \leqq u \leqq s, \\
& =(t-u) & & \text { for } \quad s \leqq u \leqq t, \\
& =(t+u) & & \text { for }-s \geqq u \geqq-t, \\
& =0 & & \text { otherwise. }
\end{aligned}
$$

We will now show that $\hat{W}_{s} /(t-s)$ is a martingale for $0 \leqq s \leqq t$. If $s_{1}<s_{2}$, then

$$
E\left(\hat{W}_{s_{2}} /\left(t-s_{2}\right)-\hat{W}_{s_{1}} /\left(t-s_{1}\right) \mid \mathscr{F}_{\left(-\infty, s_{1}\right]}^{X}\right)=E\left(W_{s_{2}} /\left(t-s_{2}\right)-W_{s_{1}} /\left(t-s_{1}\right) \mid \mathscr{F}_{\left(-\infty, s_{1}\right]}^{X}\right) .
$$

But

$$
\frac{W_{s_{2}}}{t-s_{2}}-\frac{W_{s_{1}}}{t-s_{1}}=b\left(\frac{d^{2} B}{d t^{2}}\right)\left(\frac{\psi^{s_{2}}}{t-s_{2}}-\frac{\psi^{s_{1}}}{t-s_{1}}\right)
$$

and

$$
\frac{\psi^{s_{2}}(u)}{t-s_{2}}-\frac{\psi^{s_{1}}(u)}{t-s_{1}}=0 \text { for }-s_{1} \leqq u \leqq+s_{1}
$$

Therefore, by Proposition 4.5,

$$
E\left(W_{s_{2}} /\left(t-s_{2}\right)-W_{s_{1}} /\left(t-s_{1}\right) \mid \mathscr{F}_{\left(-\infty, s_{1}\right]}^{X}\right)=0 .
$$

Hence, $\hat{W}_{s} /(t-s)$ is a martingale. Hence $\hat{W}_{s}^{2} /(t-s)^{2}$ is a submartingale. We subdivide the interval $[0, t]$ into $[0, t / 2],(t / 2,3 t / 4], \ldots$. The length of the $i$ th subinterval is $t / 2^{i}$. Let the endpoints be denoted by $t_{0}, t_{1}, \ldots$ Then $t_{i}=\left(\left(2^{i}-1\right) / 2^{i}\right) t$. Then by the submartingale inequality (Doob [1, Chapter VII, Theorem 3.2]),

$$
P\left(\sup _{t_{i-1} \leqq s \leqq t_{i}} \frac{\hat{W}_{s}^{2}}{(t-s)^{2}} \geqq \lambda\right) \leqq \frac{1}{\lambda} E\left(\frac{\hat{W}_{t_{i}}^{2}}{\left(t-t_{i}\right)^{2}}\right) .
$$

Therefore

$$
P\left(\sup _{t_{i}-1 \leqq s \leqq t_{i}} \hat{W}_{s}^{2} \geqq \lambda\left(t-t_{i-1}\right)^{2}\right) \leqq \frac{1}{\lambda} \frac{E\left(\hat{W}_{t_{i}}^{2}\right)}{\left(t-t_{i}\right)^{2}}
$$

Let $\lambda^{1}=\lambda\left(t-t_{i-1}\right)^{2}$. Then

Hence

$$
\begin{aligned}
P\left(\sup _{t_{i-1} \leqq s \leqq t_{i}} \hat{W}_{s}^{2} \geqq \lambda^{1}\right) & \leqq \frac{\left(t-t_{i-1}\right)^{2}}{\lambda^{1}} \frac{E\left(\hat{W}_{t_{i}}^{2}\right)}{\left(t-t_{i}\right)^{2}} \\
& \leqq \frac{4}{\lambda^{1}} E\left(\hat{W}_{t_{i}}^{2}\right) \quad \text { since } \frac{\left(t-t_{i-1}\right)^{2}}{\left(t-t_{i}\right)^{2}}=4
\end{aligned}
$$

$$
P\left(\sup _{0 \leqq s \leqq t} \hat{W}_{s}^{2} \geqq \lambda\right) \leqq \frac{4}{\lambda} \sum_{i=1}^{\infty} E\left(\hat{W}_{t_{i}}^{2}\right)
$$

Let

$$
\begin{aligned}
\theta^{t_{i}}(u) & \equiv\left(t-t_{i}\right), \quad-t_{i} \leqq u \leqq t_{i} \\
& \equiv\left(t-t_{i}\right)-2\left(u-t_{i}\right), \quad t_{i} \leqq u \leqq t_{i+1}, \\
& \equiv 0 \quad \text { otherwise. }
\end{aligned}
$$


Since Spt $\left(b\left(\theta^{t_{i}}-\psi^{t_{i}}\right)\right) \subset\left[t_{i}, t\right]$, Proposition 4.5 implies that

$$
\begin{aligned}
\hat{W}_{t_{i}} & =E\left(b\left(d^{2} B / d t^{2}\right)\left(\psi^{t_{i}}\right) \mid \mathscr{F}_{\left(-\infty, t_{i}\right.}^{X}\right) \\
& =E\left(b\left(d^{2} B / d t^{2}\right)\left(\theta^{t_{i}}\right) \mid \mathscr{F}_{\left(-\infty, t_{i}\right)}^{X}\right) .
\end{aligned}
$$

Therefore, by Jensen's inequality,

$$
\begin{aligned}
E\left(\hat{W}_{t_{i}}^{2}\right) & =E\left(E\left(b\left(d^{2} B / d t^{2}\right)\left(\theta^{t_{i}}\right) \mid \mathscr{F}_{\left(-\infty, t_{i}\right]}^{X}\right)^{2}\right) \\
& \leqq E\left(E\left(\left(b\left(d^{2} B / d t^{2}\right)\left(\theta^{t_{i}}\right)\right)^{2} \mid \mathscr{F}_{\left(-\infty, t_{i}\right.}^{X}\right)\right) \\
& =E\left(b\left(d^{2} B / d t^{2}\right)\left(\theta^{t_{i}}\right)\right)^{2} .
\end{aligned}
$$

But

$$
\begin{aligned}
E\left(b\left(d^{2} B / d t^{2}\right)\left(\theta^{t_{i}}\right)\right)^{2} & =E\left(\int_{-\infty}^{\infty}\left(\dot{b}(s) \theta^{t_{i}}(s)+b(s) \dot{\theta}^{t_{i}}(s)\right)^{2} d s\right) \\
& \leqq 2 E \int_{-\infty}^{\infty}\left(\dot{b}(s) \theta^{t_{i}}(s)\right)^{2} d s+2 E \int_{-\infty}^{\infty}\left(b(s) \dot{\theta}^{t_{i}}(s)\right)^{2} d s \\
& \leqq 2 E \int_{0}^{t}(b(s))^{2} d s \cdot \frac{t}{2^{i}}+8 E \int_{t_{i}}^{t_{i+1}} b^{2}(s) d s
\end{aligned}
$$

Therefore

$$
\sum_{i=1}^{\infty} E\left(\hat{W}_{t_{i}}^{2}\right) \leqq 2 t E \int_{0}^{t}(b(s))^{2} d s+8 E \int_{0}^{t} b^{2}(s) d s
$$

and

$$
P\left(\sup _{0 \leqq s \leqq t} \hat{W}_{s}^{2} \geqq \lambda\right) \leqq \frac{K}{\lambda} \int_{0}^{t}\left((b(s))^{2}+(b(s))^{2}\right) d s .
$$

The result then follows by noting that

$$
P\left(\sup _{0 \leqq s \leqq t} Y_{s}^{2} \geqq \lambda\right) \leqq P\left(\sup _{0 \leqq s \leqq t} Z_{s}^{2} \geqq \lambda / 4\right)+P\left(\sup _{0 \leqq s \leqq t} \hat{W}_{s}^{2} \geqq \lambda / 4\right)
$$

and

$$
P\left(\sup _{0 \leqq s \leqq t}\left|Y_{s}\right| \geqq \lambda\right)=P\left(\sup _{0 \leqq s \leqq t} Y_{s}^{2} \geqq \lambda^{2}\right)
$$

We also have

$$
E\left(\sup _{0 \leqq s \leqq t} Y_{s}^{2}\right) \leqq 2 E\left(\sup _{0 \leqq s \leqq t} Z_{s}^{2}\right)+2 E\left(\sup _{0 \leqq s \leqq t} \hat{W}_{s}^{2}\right)
$$

Since $Z_{s}$ is a martingale, by applying a theorem of J. L. Doob [1, Chapter VII, Theorem 3.4] to $\left|Z_{s}\right|$ we obtain

$$
E\left(\sup _{0 \leqq s \leqq t} Z_{s}^{2}\right) \leqq 4 E\left(Y_{t}^{2}\right)=K_{1}^{1} E\left[\int_{0}^{t}\left((b(s))^{2}+(b(s))^{2}\right) d s\right]
$$

Similarly,

$$
E\left(\sup _{t_{i}-1 \leqq s \leqq t_{i}} \hat{W}_{s}^{2}\right) \leqq 16 E\left(\hat{W}_{t_{i}}^{2}\right)
$$


and

$$
E\left(\sup _{0 \leqq s \leqq t} \hat{W}_{s}^{2}\right) \leqq 16 \sum_{i=1}^{\infty} E\left(\hat{W}_{t_{i}}^{2}\right)
$$

and therefore

$$
E\left(\sup _{0 \leqq s \leqq t} Y_{s}^{2}\right) \leqq K \int_{0}^{t}\left((\dot{b}(s))^{2}+(b(s))^{2}\right) d s .
$$

REMARK 5.1. Note that the proof of Lemma 5.1 is valid if $d^{2} B / d t^{2}$ is replaced by any generalized random function with independent values for which $\|\phi\|_{\Gamma} \leqq k\|\phi\|_{2,1}$. However the following corollary depends on the sample path continuity of $B$.

COROLlaRY. The sample paths of the random function $s \leadsto \int^{s} \int b(t, \omega) d^{2} B / d t^{2}$ are almost surely continuous.

Proof. Regularizing as above we can choose, for a given $t$, a sequence $b_{n} \rightarrow b$ in $\|\cdot\|_{2,1 ; t}$ such that each $b_{n}$ is almost surely $C^{\infty}$. Since the sample paths of $B$ are almost surely continuous, it is easy to verify that the sample paths of

$$
s \leadsto \int s b_{n}(t, \omega)\left(d^{2} B / d t^{2}\right) d t
$$

are almost surely continuous.

But according to Lemma 5.1,

$$
P\left(\sup _{0 \leqq s \leqq t}\left|\int^{s} \int\left(b(t, \omega)-b_{n}(t, \omega)\right) \frac{d^{2} B}{d t^{2}}\right| \geqq \lambda\right) \leqq \frac{K_{1}}{\lambda^{2}}\left\|b-b_{n}\right\|_{2,1 ; t} .
$$

Hence we can choose a subsequence $\left\{n_{k}\right\}$ such that

$$
P\left(\sup _{0 \leqq s \leqq t}\left|\int^{s} \int\left(b(t, \omega)-b_{n_{k}}(t, \omega)\right) \frac{d^{2} B}{d t^{2}}\right| \geqq \frac{\lambda}{k}\right) \leqq \frac{K_{1} k^{2}}{2^{k} \lambda^{2}}
$$

But then by the Borel-Cantelli lemma

$$
P\left(\sup _{0 \leqq s \leqq t}\left|\iint\left(b(t, \omega)-b_{n_{k}}(t, \omega)\right) \frac{d^{2} B}{d t^{2}}\right| \geqq \frac{\lambda}{k} \quad \text { for infinitely many } k\right)=0 .
$$

Hence

$$
\int^{s} \int b_{n_{k}}(t, \omega) \frac{d^{2} B}{d t^{2}} \rightarrow \iint b(t, \omega) \frac{d^{2} B}{d t^{2}} d t
$$

uniformly for $s \in[0, t]$ with probability one. Therefore $s \leadsto \int s \int b(t, \omega)\left(d^{2} B / d t^{2}\right) d t$ has continuous sample paths with probability one.

THEOREM 5.4. Assume that $c(t) \in C^{\infty} \cap H^{\infty, 1}$ and has support in $[0, \infty)$. Assume that $a$ is a progressively measurable mapping from $C^{1}$ into $\mathscr{D}_{2}^{\prime}$ where

$$
\mathscr{D}_{j}^{\prime} \equiv\left\{T: T \in \mathscr{D}^{\prime} ; T=D^{j} f, f \in C\right\}
$$


and that

$$
\left|\iint a(f)-\int^{s} \int a(g)\right| \leqq k_{t}|f(s)-g(s)| \text { for } 0 \leqq s \leqq t .
$$

Also assume that for any $f$, Spt $(a(f)) \subset[0, \infty)$. Then the stochastic differential equation

$$
d^{3} Y \mid d t^{3}=a(Y)+c(t) Y(t)\left(d^{2} B / d t^{2}\right)
$$

with past condition

$$
Y(t)=0 \text { for } t<0
$$

has a solution whose sample paths are concentrated on $C^{1}$.

Proof. If we set $Y_{0} \equiv 0$, then $Y_{1} \equiv \iiint a(0) \in C^{1}$. For $n=1,2,3, \ldots$, let

$$
Y_{n+1} \equiv \iiint\left[a\left(Y_{n}\right)+c(t) Y_{n}\left(d^{2} B / d t^{2}\right)\right] d t
$$

In order that the definition (5.13) of $Y_{n+1}$ make sense we have to check that $\left\|Y_{n}\right\|_{2,1 ; t}^{2}<\infty$ for each $t$ and that $Y_{n} \in C^{1}$ a.s. Then the corollary to Lemma 5.1 implies that $Y_{n+1}$ has $C^{1}$ sample functions a.s. It will follow from what follows below that $\left\|Y_{n+1}\right\|_{2,1 ; t}^{2}<\infty$ for each $t$ and hence the sequence $\left\{Y_{n}\right\}$ is well defined. It is also obvious that one can choose the arbitrary constants in (5.13) so that the condition (5.12b) is satisfied. We assume below that $Y_{n+1}$ is so chosen.

Now

$$
D^{3} Y_{n+1}-D^{3} Y_{n}=a\left(Y_{n}\right)-a\left(Y_{n-1}\right)+c(t)\left(Y_{n}-Y_{n-1}\right) d^{2} B / d t^{2} .
$$

If Spt $(\phi) \subset[0, t]$ then

$$
\begin{aligned}
E\left[\left(D^{3} Y_{n+1}-D^{3} Y_{n}\right)(\phi)\right]^{2} & \\
\leqq & 2 E\left[\left(a\left(Y_{n}\right)-a\left(Y_{n-1}\right)\right)(\phi)\right]^{2}+2 E\left[c(\cdot)\left(Y_{n}-Y_{n-1}\right)(\phi)\right]_{\Gamma}^{2} \\
\leqq & 2 E\left[\left(a\left(Y_{n}\right)-a\left(Y_{n-1}\right)\right)(\phi)\right]^{2}+6 E\left[\int\left(\dot{c}(t)\left(Y_{n}(t)-Y_{n-1}(t)\right) \phi(t)\right)^{2} d t\right] \\
& +6 E\left[\int\left(c(t)\left(\dot{Y}_{n}(t)-\dot{Y}_{n-1}(t)\right) \phi(t)\right)^{2} d t\right] \\
& +6 E\left[\int\left(c(t)\left(Y_{n}(t)-Y_{n-1}(t)\right) \dot{\phi}(t)\right)^{2} d t\right] \\
\leqq & 2 E\left[\left(a\left(Y_{n}\right)-a\left(Y_{n-1}\right)\right)(\phi)\right]^{2}+k_{1}\left\|Y_{n}-Y_{n-1}\right\|_{2,1 ; t}^{2}\|\phi\|_{1, \infty}^{2}
\end{aligned}
$$

for an appropriate constant $k_{1}$.

Let $\psi_{1}^{s}=\phi^{s}$ as defined in Lemma 5.1 and $\psi_{2}^{s}(u)=\int_{-\infty}^{u} \psi^{s}(u) d u$ where

$$
\begin{aligned}
\psi^{s}(u) & =\phi^{s}(u), & & -s \leqq u \leqq s, \\
& =-\phi^{s}(-2 s-u), & & -s \geqq u \geqq-3 s .
\end{aligned}
$$


Then $\operatorname{Spt}\left(\psi_{1}^{s}\right) \subset(-\infty, s]$ and $\operatorname{Spt}\left(\psi_{2}^{s}\right) \subset(-\infty, s]$. Since for each $n$, Spt $\left(D^{3} Y_{n}\right)$ $\subset[0, \infty)$, it is easy to verify that $\left(D^{3} Y_{n+1}-D^{3} Y_{n}\right)\left(\psi_{j}^{s}\right), j=1,2$, are well defined and that

$$
\iiint\left(D^{3} Y_{n+1}-D^{3} Y_{n}\right)=\left(D^{3} Y_{n+1}-D^{3} Y_{n}\right)\left(\psi_{2}^{s}\right) \quad \text { a.s. }
$$

and

$$
\int^{s} \int\left(D^{3} Y_{n+1}-D^{3} Y_{n}\right)=\left(D^{3} Y_{n+1}-D^{3} Y_{n}\right)\left(\psi_{1}^{s}\right) \text { a.s. }
$$

It then follows that

$$
E\left(Y_{n+1}(t)-Y_{n}(t)\right)^{2} \leqq 2 E\left[\iiint\left(a\left(Y_{n}\right)-a\left(Y_{n-1}\right)\right)\right]^{2}+k_{2}\left\|Y_{n}-Y_{n-1}\right\|_{2,1 ; t}^{2} .
$$

Hence by our hypotheses,

$$
E\left(Y_{n+1}(t)-Y_{n}(t)\right)^{2} \leqq k_{3} E\left[\int_{-\infty}^{t}\left(Y_{n}(s)-Y_{n-1}(s)\right)^{2} d s\right]+k_{2}\left\|Y_{n}-Y_{n-1}\right\|_{2,1 ; 1}^{2} .
$$

Therefore

$$
E\left(Y_{n+1}(t)-Y_{n}(t)\right)^{2} \leqq\left(k_{2}+k_{3}\right)\left\|Y_{n}-Y_{n-1}\right\|_{2,1 ; t \cdot}^{2}
$$

Also

Therefore for $s \leqq t$,

$$
\begin{aligned}
E\left(\dot{Y}_{n+1}(t)-\dot{Y}_{n}(t)\right)^{2} & \leqq 2 E\left[\iint a\left(Y_{n}\right)-a\left(Y_{n-1}\right)\right]^{2}+k_{4}\left\|Y_{n}-Y_{n-1}\right\|_{2,1 ; t}^{2} \\
& \leqq 2 k_{t}^{2} E\left[\left|Y_{n}(t)-Y_{n-1}(t)\right|^{2}\right]+k_{4}\left\|Y_{n}-Y_{n-1}\right\|_{2,1 ; t}^{2} \\
& \leqq 2 k_{t}^{2} E\left[\int_{-\infty}^{t}\left|\dot{Y}_{n}(t)-\dot{Y}_{n-1}(t)\right|^{2} d t\right]+k_{4}\left\|Y_{n}-Y_{n-1}\right\|_{2,1 ; t}^{2} \\
& \leqq 2 k_{t}^{2}\left\|Y_{n}-Y_{n-1}\right\|_{2,1 ; t}^{2}+k_{4}\left\|Y_{n}-Y_{n-1}\right\|_{2,1 ; t}^{2}
\end{aligned}
$$

$$
E\left(\dot{Y}_{n+1}(s)-\dot{Y}_{n}(s)\right)^{2} \leqq\left(k_{4}+k_{5}\right)\left\|Y_{n}-Y_{n-1}\right\|_{2,1 ; s \cdot}^{2}
$$

From (5.14) and (5.15) we have for $u \leqq t$,

$$
\left\|Y_{n+1}-Y_{n}\right\|_{2,1 ; u}^{2} \leqq k_{6} \int_{-\infty}^{u}\left\|Y_{n}-Y_{n-1}\right\|_{2,1 ; s}^{2} d s .
$$

Therefore for $u \leqq t$,

$$
\left\|Y_{n+1}-Y_{n}\right\|_{2,1 ; u}^{2} \leqq \frac{k_{6}^{n-1} u^{n-1}}{(n-1) !}\left\|Y_{1}-Y_{0}\right\|_{2,1 ; u}^{2}
$$

where $k_{6}$ may depend on $t$ but not on $n$.

Let $g_{n, m} \equiv\left\|Y_{n}-Y_{m}\right\|_{2,1 ; t}^{2}$ and $g_{j} \equiv g_{j, j-1}$. Then for $m<n$,

$$
\begin{aligned}
g_{n, m} & \leqq 2^{-m} \sum_{j=m+1}^{\infty} 2^{j} g_{j} \\
& \leqq 2^{-m} \sum_{j=1}^{\infty} \frac{2^{j} k_{6}^{j-1} t^{j-1}}{(j-1) !} g_{1} .
\end{aligned}
$$


Therefore $Y_{n}(\cdot)$ converges in $\|\cdot\|_{2,1 ; t}$ to $Y_{\infty}(\cdot)$ and

$$
g_{m, \infty} \leqq 2^{-m} \sum_{j=1}^{\infty} \frac{2^{j} k_{6}^{j=1} t^{j-1}}{(j-1) !} g_{1}
$$

Therefore, by Lemma 5.1,

$$
P\left(\sup _{0 \leqq s \leqq t}\left|\int^{s} \int\left(Y_{n+1}-Y_{n}\right) c(\cdot) \frac{d^{2} B}{d t^{2}}\right| \geqq \frac{1}{2^{n}}\right) \leqq \frac{4^{n} k_{6}^{n-1} t^{n-1}}{(n-1) !} g_{1} K_{1} .
$$

Since the right-hand side converges, the Borel-Cantelli lemma implies that $\int^{s} \int Y_{n} c(\cdot)\left(d^{2} B / d t^{2}\right)$ converges uniformly on $[0, t]$ with probability one. Also

$$
\begin{aligned}
P\left\{\sup _{0 \leqq s \leqq t} \mid \int^{s} \int a\left(Y_{n+1}\right)-\int^{s}\right. & \left.\int a\left(Y_{n}\right) \mid \geqq \frac{1}{2^{n}}\right\} \\
& \leqq P\left\{\sup _{0 \leqq s \leqq t} k_{t}\left|Y_{n+1}(s)-Y_{n}(s)\right| \geqq \frac{1}{2^{n}}\right\} \\
& \leqq P\left\{k_{t} \int_{-\infty}^{t}\left|\dot{Y}_{n+1}(s)-\dot{Y}_{n}(s)\right| \geqq \frac{1}{2^{n}}\right\}
\end{aligned}
$$

But by Chebyshev's inequality this becomes

$$
\begin{aligned}
P\left\{\sup _{0 \leqq s \leqq t} \mid \int^{s} \int a\left(Y_{n+1}\right)-\int^{s}\right. & \left.\int a\left(Y_{n}\right) \mid \geqq \frac{1}{2^{n}}\right\} \\
& \leqq k_{t}^{2} t^{2} 4^{n} E\left[\int_{-\infty}^{t}\left(\dot{Y}_{n+1}(s)-\dot{Y}_{n}(s)\right)^{2} d s\right] \\
& \leqq \frac{k_{t}^{2} 4^{n} k_{6}^{n-1} t^{n+1}}{(n-1) !}\left\|Y_{1}-Y_{0}\right\|_{2,1 ; t}^{2} .
\end{aligned}
$$

Since

$$
\sum_{n=1}^{\infty} \frac{k_{t}^{2} 4^{n} k_{6}^{n-1} t^{n+1}}{(n-1) !}\left\|Y_{1}-Y_{0}\right\|_{2,1 ; t}^{2}
$$

converges, the Borel-Cantelli lemma implies that $\int s a\left(Y_{n}\right)$ converges uniformly for $s \in[0, t]$ with probability one. Since by hypothesis

$$
\left|\iint a\left(Y_{\infty}\right)-a\left(Y_{n}\right)\right| \leqq k_{t}\left|Y_{\infty}(s)-Y_{n}(s)\right|, \quad 0 \leqq s \leqq t,
$$

it follows that $\int^{s} \int a\left(Y_{n}\right) \rightarrow \int^{s} \int a\left(Y_{\infty}\right)$ uniformly for $s \in[0, t]$ with probability one.

To complete the proof that $Y_{\infty}$ satisfies equation 5.12, it suffices to show that

$$
\int^{s} \int c(\cdot) Y_{n} \frac{d^{2} B}{d t^{2}} \rightarrow \int^{s} \int c(\cdot) Y_{\infty} \frac{d^{2} B}{d t^{2}}
$$

uniformly for $s \in[0, t]$ with probability one. 
According to Lemma 5.1

$$
\begin{aligned}
P\left(\sup _{0 \leqq s \leqq t} \mid \int^{s} \int c(t)\left(Y_{n}(t)-Y_{\infty}(t)\right)\right. & \left.\frac{d^{2} B}{d t^{2}} d t \mid \geqq \begin{array}{l}
1 \\
n
\end{array}\right) \\
& \leqq n^{2} K_{1}\left\|c(t)\left(Y_{n}(t)-Y_{\infty}(t)\right)\right\|_{2,1 ; t}^{2} \\
& \leqq n^{2} k_{7} 2^{-n} \sum_{j=1}^{\infty} \frac{k_{6}^{j-1} t^{j-1} 2^{j}}{(j-1) !} g_{1}
\end{aligned}
$$

where $k_{7}$ is a constant. Since $\sum_{n=1}^{\infty} n^{2} 2^{-n}<\infty$, the Borel-Cantelli lemma implies that

$$
\iint c(t) Y_{n}(t) \frac{d^{2} B}{d t^{2}} d t \rightarrow \iint c(t) Y_{\infty}(t) \frac{d^{2} B}{d t^{2}} d t
$$

uniformly for $s \in[0, t]$ with probability one.

REMARK 5.2. It is important to note that in contrast to the case of Itô equations the above methods do not easily extend to the general nonlinear equation

$$
d^{3} Y / d t^{3}=a(Y)+c(t) g(Y(t)) d^{2} B / d t^{2}
$$

where $g$ is a real valued function. The reason for this is that to follow up the analogous type of reasoning to that used for Itô equations would require that we restrict ourselves to functions $g$ which satisfy:

$$
\begin{aligned}
&\left|g\left(Y_{1}(t)\right)-g\left(Y_{2}(t)\right)\right|+\left|d\left(g\left(Y_{1}(t)\right)-g\left(Y_{2}(t)\right)\right) / d t\right| \\
& \leqq k_{1}\left|Y_{1}(t)-Y_{2}(t)\right|+k_{2}\left|\dot{Y}_{1}(t)-\dot{Y}_{2}(t)\right| .
\end{aligned}
$$

But

$$
\begin{aligned}
\mid d(g( & \left.\left.Y_{1}(t)\right)-g\left(Y_{2}(t)\right)\right) / d t \mid \\
\quad & =\left|\dot{g}\left(Y_{1}(t)\right) \dot{Y}_{1}(t)-g\left(Y_{2}(t)\right) \dot{Y}_{2}(t)\right| \\
\quad & =\left|\left(\dot{g}\left(Y_{1}(t)\right) \dot{Y}_{1}(t)-\dot{g}\left(Y_{1}(t)\right) \dot{Y}_{2}(t)\right)+\left(\dot{g}\left(Y_{1}(t)\right) \dot{Y}_{2}(t)-\dot{g}\left(Y_{2}(t)\right) \dot{Y}_{2}(t)\right)\right| \\
& \geqq|| \dot{g}\left(Y_{1}(t)\right)\left(\dot{Y}_{1}(t)-\dot{Y}_{2}(t)\right)|-| \dot{Y}_{2}(t)\left(\dot{g}\left(Y_{1}(t)\right)-\dot{g}\left(Y_{2}(t)\right)|| .\right.
\end{aligned}
$$

But if for any $Y_{1}(t) \neq Y_{2}(t), \dot{g}\left(Y_{1}(t)\right) \neq \dot{g}\left(Y_{2}(t)\right)$, then $\left|\dot{Y}_{2}\left(\dot{g}\left(Y_{1}(t)\right)-\dot{g}\left(Y_{2}(t)\right)\right)\right|$ can be made arbitrarily large under the constraints $\left|Y_{1}(t)-Y_{2}(t)\right|+\left|\dot{Y}_{1}(t)-\dot{Y}_{2}(t)\right|<K$. Therefore the only functions $g$ satisfying (5.17) are those such that $\dot{g}(s)=c$ or $g(s)=c s+b$ where $b$ and $c$ are constants. In view of this, it appears that to prove existence for equations of the type (5.16) different methods are needed. Such existence theorems will be proved elsewhere.

6. Markov properties of solutions of stochastic equations. A generalized process $Y$ is called a local process with respect to $\left\{\mathscr{F}_{(-\infty, t]}: t \in R^{1}\right\}$ if for $t \in R^{1}, \varepsilon>0$, $A \in \mathscr{F}_{(-\infty, t-\varepsilon]}, B \in \mathscr{F}_{[t+\varepsilon, \infty)}$, then $P\left(A \cap B \mid \mathscr{F}_{t}^{*}\right)=P\left(A \mid \mathscr{F}_{t}^{*}\right) \cdot P\left(B \mid \mathscr{F}_{t}^{*}\right)$ a.s. where $\mathscr{F}_{t}^{*} \equiv \bigcap_{\varepsilon>0} \mathscr{F}_{(t-\varepsilon, t+\varepsilon)}$ (cf. Urbanik [21]).

A generalized process is weakly Markov of order $N$ with respect to the family $\left\{\mathscr{F}_{(-\infty, t]}: t \in R^{1}\right\}$ if there exist $N$ random functions $Z_{1}^{t}, \ldots, Z_{N}^{t}$, such that for each 
$t$ and $i, Z_{i}^{t}$ is measurable with respect to $\mathscr{F}_{(-\infty, t]}$ and such that if $A \in \mathscr{F}_{(-\infty, t]}$, $B \in \mathscr{F}_{[t, \infty)}$, then

$$
P\left(A \cap B \mid Z_{1}^{t}, \ldots, Z_{N}^{t}\right)=P\left(A \mid Z_{1}^{t}, \ldots, Z_{N}^{t}\right) \cdot P\left(B \mid Z_{1}^{t}, \ldots, Z_{N}^{t}\right) \quad \text { a.s. }
$$

(cf. Hida [6]).

A mapping $\Phi: \mathscr{D}^{\prime} \rightarrow \mathscr{D}^{\prime}$ is a local mapping if for $T_{1}, T_{2} \in \mathscr{D}^{\prime}$, and any open interval $U$, the property

$$
T_{1}(\phi)=T_{2}(\phi) \text { for every } \phi \text { with } \operatorname{Spt}(\phi) \subset U,
$$

implies that

$$
\Phi T_{1}(\phi)=\Phi T_{2}(\phi) \text { for every } \phi \text { with Spt }(\phi) \subset U .
$$

THeOREM 6.1. If $X$ is a generalized process with independent values, $\operatorname{Spt}(X) \subset[0, \infty)$, $T$ is a continuous, linear, local transformation on $\mathscr{D}^{\prime}$ and if the solution of $T f=g$ are analytic in the complement of $\operatorname{Spt}(g)$, then the solution of $T Y=X, Y(\phi)=0$ if Spt $(\phi) \subset(-\infty, 0]$, is a local process with respect to $\left\{\mathscr{F}_{(-\infty, t]}^{Y}\right\} .\left(\mathscr{F}_{(-\infty, t]}^{Y}\right.$ is the $\sigma$-field generated by the random variables $Y(\phi), \phi \in \mathscr{D}, \operatorname{Spt}(\phi) \subset(-\infty, t]$.)

Proof. Let $0 \leqq s<t<u_{1}<u_{2}<u_{3}<v<w$. Let $A \in \mathscr{F}_{(s, t)}^{Y}$ and $B \in \mathscr{F}_{(v, w)}^{Y}$. Then it suffices to show that

$$
P\left(A \cap B \mid \mathscr{F}_{\left(u_{1}, u_{3}\right)}^{Y}\right)=P\left(A \mid \mathscr{F}_{\left(u_{1}, u_{3}\right)}^{Y}\right) \cdot P\left(B \mid \mathscr{F}_{\left(u_{1}, u_{3}\right)}^{Y}\right) \quad \text { a.s. }
$$

It therefore suffices to show that if $B=\left\{Y\left(\phi_{1}\right) \in B_{1}, \ldots, Y\left(\phi_{n}\right) \in B_{n}\right\}, \phi_{1}, \ldots, \phi_{n} \in \mathscr{D}$, $B_{1}, \ldots, B_{n} \in \mathscr{B}\left(R_{1}\right)$, Spt $\left(\phi_{i}\right) \subset(v, w)$, then

$$
P\left(B \mid \mathscr{F}_{\left(u_{1}, u_{3}\right)}^{Y}\right)=P\left(B \mid \mathscr{F}_{\left(s, u_{3}\right)}^{Y}\right) \text { a.s. }
$$

Let $X=X \psi_{1}+X \psi_{2}$ where $\psi_{1}, \psi_{2}$ are $C^{\infty}$ and $\operatorname{Spt}\left(\dot{\psi}_{i}\right) \subset\left(u_{1}, u_{2}\right), i=1,2, \operatorname{Spt}\left(\psi_{1}\right)$ $\subset\left(-\infty, u_{2}\right]$, Spt $\left(\psi_{2}\right) \subset\left[u_{1}, \infty\right)$. Then in $\left[u_{2}, \infty\right)$,

$$
T Y=X \psi_{2} .
$$

Let $Y_{2}$ be a particular solution of (6.1). Note that conditioned on $\mathscr{F}_{\left[u_{1}, \infty\right)}^{Y}, Y_{2}$ is independent of $\mathscr{F}_{\left(s, u_{1}\right)}^{Y}$; in fact, since $T$ is local, $Y_{2}$ is measurable with respect to $\mathscr{F}_{\left[u_{1}, \infty\right)}^{Y}$. Then $Y=Y_{1}+Y_{2}$ where $Y_{1}$ is a solution of $T Y=X \psi_{1}$. But $Y_{1}$ is analytic in $\left[u_{2}, \infty\right)$ and therefore is uniquely determined by its restriction to $\left(u_{2}, u_{3}\right)$. Hence the restriction of $Y_{1}$ to $(v, w)$ is measurable with respect to the $\sigma$-field generated by the restrictions of $Y$ and $Y_{2}$ to $\left[u_{1}, \infty\right)$. Therefore

$$
P\left(Y\left(\phi_{1}\right) \in B_{1}, \ldots, Y\left(\phi_{n}\right) \in B_{n} \mid \mathscr{F}_{\left(s, u_{3}\right)}^{Y}\right)=P\left(Y\left(\phi_{1}\right) \in B_{1}, \ldots, Y\left(\phi_{n}\right) \in B_{n} \mid \mathscr{F}_{\left(u_{1}, u_{3}\right)}^{Y}\right)
$$

since we have shown that conditioned on $Y$ restricted to $\left(u_{1}, u_{3}\right), Y$ restricted to $(v, w)$ is independent of $\mathscr{F}_{\left(s, u_{1}\right)}^{Y}$. Hence the theorem is proved.

COROLlary. If $P(D)$ is a polynomial in the differentiation operator $D$ and if $X$ is a generalized process, then the solution of $P(D) Y=X$ is $\mathscr{F}^{Y}$-local. 
THEOREM 6.2. If $X$ is the nth derivative of an ordinary stochastic process $\hat{X}$ with independent increments, $\operatorname{Spt}(\hat{X}) \subset[0, \infty)$ and $m>n$, then the solution of the equation

$$
D^{m} Y+a_{1} D^{m-1} Y+\cdots+a_{m} Y=X
$$

with past condition $Y(\phi)=0$ if $\operatorname{Spt}(\phi) \subset(-\infty, 0]$ is weakly Markov of finite order with respect to $\left\{\mathscr{F}_{(-\infty, t]}^{Y} ; t \in R^{1}\right\}$.

Proof. Equation 6.2 can be transformed into an equation of the form

$$
P(D)\left(Y-\sum_{i=-m}^{m} c_{i} \hat{X}^{(i)}\right)=\sum_{i=-m}^{0} b_{i} \hat{X}^{(i)}
$$

where $c_{i}$ 's and $b_{i}$ 's are constants and

$$
\begin{aligned}
\hat{X}^{(i)} & =\frac{d^{i} \hat{X}}{d t^{i}} \text { if } i \geqq 0, \\
& =\int \ldots \int \hat{X} \text {, the } i \text {-fold indefinite integral if } i<0 .
\end{aligned}
$$

The right-hand side of (6.3) is an ordinary function $f(t)$ and we can solve the equations $P(D) Z=f$ in the usual way. If for example, $P(D)=\prod_{i=1}^{m}\left(D-p_{i}\right)$ with the $p_{i}$ 's all distinct, then

$$
Z(t)=\sum_{i=0}^{m} d_{i} e^{p_{i} t} \int_{0}^{t} e^{-p_{i} t} f(t) d t .
$$

It is then easy to verify that $Y=\sum_{i=-m}^{m} c_{i} \hat{X}^{(i)}+Z(t)$ is weakly Markov of finite order with respect to $\left\{\mathscr{F}_{(-\infty, t]}^{Y}\right\}$ with splitting variables $\int_{-\infty}^{t} e^{-p_{i} t} f(t) d t, i=1, \ldots, m$ and $\hat{X}^{(i)},-m \leqq i \leqq 0$.

Examples. 6.1. $\left(D^{2}-1\right) Y=c D^{2} B, c \in C^{\infty}$, Spt $(c) \subset[0, \infty), B=$ Brownian motion. Then

$$
Y(t)=X(t)+\frac{1}{2} e^{t} \int_{0}^{t} e^{-s} X(s) d s-\frac{1}{2} e^{-t} \int_{0}^{t} e^{s} X(s) d s
$$

where $X(s)=\int^{s} \int c D^{2} B . \quad Y(t)$ is weakly Markov of finite order with respect to $\left\{\mathscr{F}_{(-\infty, t]}^{Y} ; t \in R^{1}\right\}$ and is also local with respect to $\left\{\mathscr{F}_{(-\infty, t]}^{Y} ; t \in R^{1}\right\}$.

6.2. $\left(D^{2}-1\right) Y=c D^{3} B$, with $c$ as in 6.1. Then

$$
Y(t)=\dot{X}+\frac{1}{2} e^{t} \int_{0}^{t} e^{-s} X(s) d s+\frac{1}{2} e^{-t} \int_{0}^{t} e^{s} X(s) d s
$$

where $X(s)=\int^{s} \iint c D^{3} B$. This is an example of a weakly Markov process of finite order which is not an ordinary process.

6.3. $D Y=c\left(D B+D^{2} B\right)$, with $c$ as above. Then $Y$ is weakly Markov of finite order and local with respect to $\left\{\mathscr{F}_{(-\infty, t]}^{Y}\right\}$.

THEOREM 6.3. The only local mapping $\Phi$ from $C[0,1]$ to $C^{1}[0,1]$ is the constant mapping. 
Proof. We first show that $\Phi(f)\left(x_{0}\right)$ must be a function of $f\left(x_{0}\right)$ for fixed $x_{0}$. If not, then there are two functions $f_{1}(\cdot)$ and $f_{2}(\cdot)$ such that $f_{1}\left(x_{0}\right)=f_{2}\left(x_{0}\right)$ but $\Phi\left(f_{1}\right)\left(x_{0}\right) \neq \Phi\left(f_{2}\right)\left(x_{0}\right)$

Then define

But since $\Phi$ is local,

$$
\begin{aligned}
f_{3}(x) & =f_{1}(x), \quad x \leqq x_{0} \\
& =f_{2}(x), \quad x>x_{0} .
\end{aligned}
$$

$$
\begin{aligned}
\Phi\left(f_{3}\right)(x) & =\Phi\left(f_{1}\right)(x) \text { for } x<x_{0}, \\
& =\Phi\left(f_{2}\right)(x) \text { for } x>x_{0} .
\end{aligned}
$$

But since $\Phi(f)$ must be continuous, $\Phi\left(f_{1}\right)\left(x_{0}\right)=\Phi\left(f_{3}\right)\left(x_{0}\right)=\Phi\left(f_{2}\right)\left(x_{0}\right)$ which yields contradiction. Hence $\Phi(f)(x)=F(x, f(x))$.

Since $(d / d x)(\Phi(f))(x)$ is continuous in $x$ for each $f$ and is local, the same reasoning implies that it is a function of $x$ and $f(x)$, say $G(x, f(x))$. But then $F(x, f(x))$ $=\int_{0}^{x} G(y, f(y)) d y$.

We now show that $G(x, y)$ is bounded on a set of the form $[0,1] \times\left[-y_{1}, y_{1}\right]$. If not, let $\left(x_{i}, y_{i}\right)$ be a sequence of points such that $G\left(x_{i}, y_{i}\right) \rightarrow \infty$. Since $[0,1] \times\left[-y_{1}, y_{1}\right]$ is compact, there is no loss of generality in assuming that $\left(x_{i}, y_{i}\right) \rightarrow\left(x_{0}, y_{0}\right)$. Furthermore since for each $y, G(x, y)$ is continuous in $x$, there is no loss of generality in assuming that the $x_{i}$ are all distinct. But then there exists a piecewise linear function $f(x)$ for which $G(x, f(x))$ is unbounded. But this contradicts the fact that $G(x, f(x))$ is continuous on $[0,1]$. Hence $G$ is bounded on the set $[0,1] \times\left[-y_{1}, y_{1}\right]$.

We next show that for each $x_{0}, F\left(x_{0}, y\right)$ is constant in $y$. Let $y_{1}<y_{2}$. Noting that

$$
F\left(x_{0}, y\right)=\int_{0}^{x_{0}} G(x, f(x)) d x
$$

for any $f(x)$ such that $f\left(x_{0}\right)=y$ we have

$$
F\left(x_{0}, y_{1}\right)=\int_{0}^{x_{0}} G\left(x, y_{1}\right) d x
$$

and for any $\varepsilon>0$,

$$
F\left(x_{0}, y_{2}\right)=\int_{0}^{x_{0}-\varepsilon} G\left(x, y_{1}\right) d x+\int_{x_{0}-\varepsilon}^{x_{0}} G\left(x, y_{1}+\frac{\left(y_{2}-y_{1}\right)}{\varepsilon}\left(x-x_{0}+\varepsilon\right)\right) d x .
$$

Let $M$ be the bound of $|G(x, y)|$ on $[0,1] \times[-R ; R]$ where $R=\max \left(\left|y_{1}\right|,\left|y_{2}\right|\right)$. Then $\left|F\left(x_{0}, y_{1}\right)-F\left(x_{0}, y_{2}\right)\right| \leqq 2 \varepsilon M$. Hence $F\left(x_{0}, y_{1}\right)=F\left(x_{0}, y_{2}\right)$ which implies that $\Phi(f)(x)$ is a constant function independent of $f$.

Theorem 6.3 shows that the coefficient $b(\cdot)$ considered in Theorem 5.2 cannot in general be local. However, it may be that some derivative of $b(\cdot)$ is $\mathscr{F}^{\mathrm{Y}}$-local. $b^{(n)}$ is $\mathscr{F}_{Y^{-}}$local if for every interval $(\alpha, \mathscr{B}), \mathscr{F}_{(\alpha, \mathscr{B})}^{(b(Y)(n)} \subset \mathscr{F}_{(\alpha, \mathscr{B})}^{Y}$.

THEOREM 6.4. If $X$ is a generalized process with independent values and for some $m$ and $n, a^{(M)}$ and $b^{(n)}$ are $\mathscr{F}^{Y}$-local and is a solution, $Y$, of the equation 
$Y=a(Y)+b(Y) X$ exists, then the vector generalized process $(Y, a, b)$ is local with respect to $\left\{\mathscr{F}_{(-\infty, i, i]}^{X, b}\right\}$.

Proof. Let $0<u_{1}<u_{2}<u_{3}<v<w$. It suffices to show that for $B \in \mathscr{F}_{(v, w)}^{Y, a, b}$,

$$
P\left(B \mid \mathscr{F}_{\left(u_{1}, u_{3}\right)}^{X, Y, b}\right)=P\left(B \mid \mathscr{F}_{\left(-\infty, u_{3}\right)}^{X, Y, a, b}\right) \quad \text { a.s. }
$$

Let $\psi \in C^{\infty}\left(R^{1}\right)$ such that Spt $(\dot{\psi}) \subset\left(u_{1}, u_{2}\right)$, Spt $(\psi) \subset\left(u_{1}, \infty\right)$ and $\psi(t)=1$ for $t>u_{2}$. Therefore by our hypothesis it follows that if $\operatorname{Spt}(\phi) \subset\left(u_{2}, w\right)$ then

$$
a(Y)(\phi)=a(\psi Y)(\phi)+\int_{-\infty}^{\infty} p(t) \phi(t) d t
$$

where $p(\cdot)$ is a polynomial of degree $(m-1)$ and

$$
b(Y)(\phi)=b(\psi Y)(\phi)+\int_{-\infty}^{\infty} q(t) \phi(t) d t
$$

where $q(\cdot)$ is a polynomial of degree $(n-1)$.

But $p(t)$ and $q(t)$ are determined by the set $\{b(Y)(\phi), a(Y)(\phi), a(\psi Y)(\phi), b(\psi Y)(\phi)$, $\phi \in \mathscr{D}$, Spt $\left.(\phi) \subset\left(u_{2}, u_{3}\right)\right\}$. Hence conditioned on $\mathscr{F}_{\left(u_{1}, u_{3}\right)}^{X, Y, a, b}, a(Y)(\phi)$ and $b(Y)(\phi)$, are independent of $\mathscr{F}_{\left(-\infty, u_{1}\right]}^{X, Y, a, b}$ if $\operatorname{Spt}(\phi) \subset\left[u_{3}, \infty\right)$. But since $\left.X\right|_{\left(u_{2}, \infty\right)}$ is independent of $\left.Y\right|_{\left(-\infty, u_{1}\right]}$, this implies that the set of random variables

$$
\left\{a(Y)\left(\phi_{i}\right)+b(Y) X\left(\phi_{i}\right) ; i=1, \ldots, M\right\}
$$

conditioned on $\mathscr{F}_{\left(u_{1}, u_{3}\right)}^{X, Y, a, b}$ is independent of $\mathscr{F}_{\left(-\infty, w_{1}\right]}^{X, Y, a, b}$ if $\operatorname{Spt}\left(\phi_{i}\right) \subset(v, w), i=1, \ldots, M$. This implies that if $B \in \mathscr{F}_{\substack{(v, w) \\ \text {, }}, b, \text { then }}$

$$
P\left(B \mid \mathscr{F}_{\left(u_{1}, u_{3}\right)}^{X, a, b)}\right)=P\left(B \mid \mathscr{F}_{\left(-\infty, u_{3}\right)}^{X, a, b}\right) .
$$

EXAMPLE 6.4. Theorem 6.4 implies that the solution $Y$ of the equation $d^{3} Y / d t^{3}$ $=f(t)\left(d^{2} Y / d t^{2}\right)+Y\left(d^{2} B / d t^{2}\right)$ is $\mathscr{F}^{Y, B}$-local.

REMARKS. 6.1. It is easy to verify that the stochastic process $Y(t)$ of Example 6.1 induces a probability measure $P$ on the measure space $\left(C^{+}, \mathscr{B}\left(C^{+}\right)\right)$where $C^{+}$is the set of continuous functions on $R^{1}$ which are identically zero on $(-\infty, 0]$ and $\mathscr{B}\left(C^{+}\right)$is the set of Borel subsets of $C^{+}$. Theorem 6.2 implies that $Y(t)$ is weakly Markov of finite order and Theorem 6.1 implies that $Y(t)$ is a local process. In fact equation 6.4 implies that

$$
\begin{aligned}
Y(t+h)= & \frac{1}{2}\left(e^{h}+e^{-h}\right) Y(t)+\frac{1}{4}\left(e^{h}-e^{-h}\right)\left(\int_{0}^{t} e^{t-s} X(s) d s+\int_{0}^{t} e^{-(t-s)} X(s) d s\right) \\
& +\frac{1}{2} e^{t+h} \int_{t}^{t+h} e^{-s}(X(s)-X(t)) d s-\frac{1}{2} e^{-(t+h)} \int_{t}^{t+h} e^{s}(X(s)-X(t)) d s \\
& +(X(t+h)-X(t))
\end{aligned}
$$

where $X(s)=\int^{s} \int c D^{2} B$. If

$$
W(t) \equiv \int_{0}^{t} e^{t-s} X(s) d s+\int_{0}^{t} e^{-(t-s)} X(s) d s,
$$


then $(Y(t), W(t))$ is a Markov process with values in $R^{2}$, that is, $Y(t)$ is weakly Markov of order 2. Moreover for each $t, W(t)$ is $\mathscr{F}_{(-\infty, t]}^{Y}$-measurable. Let

$$
\left(\mathscr{F}_{t}^{Y *}\right)^{c}=\left\{A: A \in \mathscr{B}\left(C^{+}\right), \exists A_{1} \in \mathscr{F}_{t}^{Y *} \text { such that } P\left(A \Delta A_{1}\right)=0\right\} .
$$

Since $Y(t)$ is a local process with respect to $\left\{\mathscr{F}_{(-\infty, t]}^{Y}: t \in R^{1}\right\}, W(t)$ is $\left(\mathscr{F}_{t}^{Y *}\right)^{c}$ measurable for each $t$. One might expect that there exists a local mapping $\Phi$ on $C^{+}$ such that $W=\Phi(Y)$. However since $W(\cdot) \in C^{1}$, this would yield a contradiction to Theorem 6.3 and therefore no such mapping can exist. In fact since $Y(t)$ is a.s. nondifferentiable, it is more reasonable to conjecture that there does not even exist a local mapping $\Phi$ such that $W=\Phi(Y)$ a.s. (Although we do not attempt to prove this statement here, it is made plausible by the fact that Theorem 6.3 remains true if $C[0,1]$ is replaced by any sufficiently rich subset.) Essentially this means that the splitting information is hidden by a noise effect in the local $\sigma$-fields.

6.2. It may be that the distribution semigroups introduced by J. L. Lions [12] will be useful in the study of processes such as those considered in the above examples.

\section{REFERENCES}

1. J. L. Doob, Stochastic processes, Wiley, New York and Chapman \& Hall, London, 1953. MR 15, 445.

2. R. M. Dudley, Sub-stationary processes, Pacific J. Math. 20 (1967), 207-215. MR 34 \#8446.

3. X. Fernique, Processus linéaires, processus généralisés, Ann. Inst. Fourier (Grenoble) 17 (1967), no. 1, 1-92. MR 36 \#4628.

4. A. Friedman, Generalized functions and partial differential equations, Prentice-Hall, Englewood Cliffs, N. J., 1963. MR 29 \#2672.

5. I. M. Gel'fand and N. Ja. Vilenkin, Generalized functions, Vol. 4: Some applications of harmonic analysis, Fizmatgiz, Moscow, 1961; English transl., Academic Press, New York, 1964. MR 26 \#4173; MR 30 \#4152.

6. T. Hida, Canonical representations of Gaussian processes and their applications, Mem. Coll. Sci. Univ. Kyoto Ser. A Math. 33 (1960/61), 109-155. MR 22 \#10012.

7. K. Itô, On stochastic differential equations, Mem. Amer. Math. Soc. No. 4 (1951). MR 12, 724.

8. - On a formula concerning stochastic differentials, Nagoya Math. J. 3 (1951), 55-65. MR 13 \#363.

9. K. Itô and M. Nisio, On stationary solutions of a stochastic differential equation, J. Math. Kyoto Univ. 4 (1964), 1-75. MR 31 \#1719.

10. M. Jirina, Conditional probabilities on strictly separable o-algebras, Czechoslovak Math. J. 4(79) (1954), 372-380. (Russian) MR 16, 1034.

11. P. Lévy, Random functions: General theory with special reference to Laplacian random functions, Univ. California Publ. Statist. 1 (1953), 331-390. MR 14, 1099.

12. J. L. Lions, Les semigroupes distributions, Portugal. Math. 19 (1960), 141-164. MR 26 \#611.

13. P. A. Meyer, Intégrales stochastiques. I, II, Séminaire de Probabilités (Univ. Strasbourg, 1966/67); vol. I, Springer, Berlin, 1967, pp. 72-117. MR 37 \#7000.

14. Ju. V. Prohorov, The method of characteristic functionals, Proc. Fourth Berkeley Sympos. Math. Stat. and Prob., vol. II (1960), Univ. of California Press, Berkeley, Calif., 1961. MR 24 \#A3670. 
15. L. Schwartz, Sur l'impossibilité de la multiplication des distributions, C. R. Acad. Sci. Paris 239 (1954), 847-848. MR 16, 265.

16. ——, Théorie des distributions, Publ. Inst. Math. Univ. Strasbourg, nos. 9, 10, Hermann, Paris, 1966. MR 35 \#730.

17. —_, Radon measures on general topological spaces, Tata Institute of Fundamental Research, (to appear).

18. A. V. Skorohod, Studies in the theory of random processes, Iydat Kiev. Univ., Kiev, 1961; English transl., Addison-Wesley, Reading, Mass., 1965. MR 32 \#3082a,b.

19. J. L. Strand, Generalized stochastic ordinary differential equations, Notices Amer. Math. Soc. 15 (1968), Abstract \#654-21, p. 342.

20. F. Treves, Topological vector spaces, distributions and kernels, Academic Press, New York, 1967. MR 37 \#726.

21. K. Urbanik, Generalized stationary processes of Markovian character, Studia Math. 21 (1961/62), 261-282. MR 27 \#821.

22. K. Yosida, Functional analysis, Die Grundlehren der math. Wissenschaften, Band 123, Academic Press, New York and Springer-Verlag, Berlin, 1965. MR 31 \#5054.

MCGILl UnIVERSITY,

Montreal, Canada 